\title{
Baroclinic instability of time-dependent currents
}

\author{
By JOSEPH PEDLOSK Y ${ }^{1} \uparrow$ AND JIM THOMSON ${ }^{2}$ \\ ${ }^{1}$ Woods Hole Oceanographic Institution, Woods Hole, MA 02543, USA \\ ${ }^{2}$ MIT/WHOI Joint Program, Woods Hole, MA 02543, USA
}

(Received 18 September 2002 and in revised form 26 March 2003)

The baroclinic instability of a zonal current on the beta-plane is studied in the context of the two-layer model when the shear of the basic current is a periodic function of time. The basic shear is contained in a zonal channel and is independent of the meridional direction. The instability properties are studied in the neighbourhood of the classical steady-shear threshold for marginal stability. It is shown that the linear problem shares common features with the behaviour of the well-known Mathieu equation. That is, the oscillatory nature of the shear tends to stabilize an otherwise unstable current while, on the contrary, the oscillation is able to destabilize a current whose time-averaged shear is stable. Indeed, this parametric instability can destabilize a flow that at every instant possesses a shear that is subcritical with respect to the standard stability threshold. This is a new source of growing disturbances. The nonlinear problem is studied in the same near neighbourhood of the marginal curve. When the time-averaged flow is unstable, the presence of the oscillation in the shear produces both periodic finite-amplitude motions and aperiodic behaviour. Generally speaking, the aperiodic behaviour appears when the amplitude of the oscillating shear exceeds a critical value depending on frequency and dissipation. When the time-averaged flow is stable, i.e. subcritical, finite-amplitude aperiodic motion occurs when the amplitude of the oscillating part of the shear is large enough to lift the flow into the unstable domain for at least part of the cycle of oscillation. A particularly interesting phenomenon occurs when the time-averaged flow is stable and the oscillating part is too small to ever render the flow unstable according to the standard criteria. Nevertheless, in this regime parametric instability occurs for ranges of frequency that expand as the amplitude of the oscillating shear increases. The amplitude of the resulting unstable wave is a function of frequency and the magnitude of the oscillating shear. For some ranges of shear amplitude and oscillation frequency there exist multiple solutions. It is suggested that the nature of the response of the finite-amplitude behaviour of the baroclinic waves in the presence of the oscillating mean flow may be indicative of the role of seasonal variability in shaping eddy activity in both the atmosphere and the ocean.

\section{Introduction}

The theory of the baroclinic instability of zonal flows has, by now, a long history and the results of decades of investigation have placed the standard theory on firm ground. Conditions for instability, bounds on growth rates, and phase speeds of unstable modes have been specified and even finite-amplitude theory has been

$\dagger$ Author to whom correspondence should be addressed: jpedlosky@whoi.edu 
developed. A review of some aspects of the standard problem can be found in several texts, e.g. Pedlosky (1987).

Nevertheless, even apparently minor variations on the basic problem can produce substantial alterations in the stability problem and consequently new features in the dynamics of the wave and eddy activity that such instabilities produce. In the present paper we study perhaps the simplest version of one such alteration, the instability of time-dependent baroclinic flow. Such time dependence occurs naturally on many time scales, e.g. tidal, seasonal and at scales of the internal dynamics of the extended system in which the instability process is a component.

We consider the standard two-layer model in its simplest form in which the basic shear is independent of the meridional coordinate, $y$, on the beta-plane, i.e. the Phillips (1954) model. The additional feature on which we concentrate is the role of time variation in the shear of the basic state. The acceleration of the basic flow naturally requires an implicit forcing of the basic zonal state. It is not necessary to consider that forcing explicitly, only its resultant effect on the stability properties of the current. We will also idealize the time dependence of the flow as a periodic oscillation of the current's vertical shear but, as we discuss below, that idealized periodicity of the current to some extent can be relaxed without altering significantly the nature of our results.

There are three major consequences of the presence of the oscillating mean flows on the stability problem. First, the oscillating shear can give rise, within linear theory, to parametric instability of a flow that at each instant of time has a shear below the critical value for instability according to standard theory. This allows the development of self-excited baroclinic waves for shears normally considered stable. Similarly, the oscillation can stabilize otherwise unstable shears. Second, the finiteamplitude theory gives rise to aperiodic behaviour in cases for which the steady shear formulation always yields an eventual steady solution. Third, and most interestingly, the parametric instability can give rise to multiple solutions in the parameter range below the standard threshold and a consequent hysteresis in the amplitude response as a function of frequency.

In $\S 2$ we discuss the formulation of the linear problem and describe some of its salient features. We do this in the case in which the oscillation of the mean flow occurs on a fairly rapid time scale, of the same order as the advective time of the flow. Since the stability properties can be shown to be more sensitive to the oscillation of the mean shear when the frequency is lower, of the order of the growth rate, we then restrict attention to such frequency ranges and derive a governing linear equation for the instability which is identical to the classical Mathieu equation, (see, for example, Morse \& Feshbach 1953). The well-known results of the study of that classical equation are described in $\S 2$. Section 3 describes the development of the finiteamplitude theory for mean shears that are nearly critical according to the classical theory for time-independent flows. The nonlinear theory is developed for a form of dissipation which guarantees that the steady flow problem will yield disturbances that eventually equilibrate to steady wave amplitudes. Therefore, the resulting periodic and aperiodic behaviour that we subsequently find can be attributed unequivocally to the oscillation of the mean shear. Section 4 discusses the nonlinear theory when the timeaveraged shear is slightly supercritical and its oscillating part renders it stable for part of the cycle of the mean shear. A preliminary attempt is made to address the question of the parameter space in which various forms of wave amplitude behaviour are observed but the fact that the problem depends fundamentally on four independent parameters (amplitude of mean shear, amplitude of oscillating shear, frequency of 
oscillation, and the size of the dissipation) renders a compete census of parameter space practically impossible.

Section 5 describes the nonlinear case in which the mean shear is stable and the oscillation, if strong enough, can produce time intervals of instability. In this case the flow, although stable in the mean, becomes episodically unstable and for small enough dissipation becomes aperiodic for a range of frequencies of the mean shear's oscillation. Section 6 describes perhaps the most unusual of the cases discussed. In this section we treat shears in which both the mean shear and the instantaneous shear are always subcritical with respect to the classical shear threshold for instability. The possibility for parametric instability destabilizes these weak shears and the resulting amplitude behaviour is described both numerically and analytically. It is shown that, as a function of frequency, there are ranges in which two solutions are possible. Either a large, finite-amplitude wave is possible for some initial conditions, or the final state consists of a wave-free state for other initial conditions for the same parameter settings. The hysteresis, which follows from this behaviour, is described.

Section 7 summarizes and discusses our results. We are aware of the many idealizations of our treatment of the problem but we have, we think naturally, considered the cycle of the imposed shear as a type of seasonality within the restricted dynamics of our system and we believe that the behaviour we describe here is suggestive of the role that seasonality may play in shaping the degree of eddy activity and behaviour in more complex systems like the atmosphere and ocean. For example, a recent study by Qiu (1999) examined the stability of the North Pacific Subtropical Countercurrent. As shown in that paper there is a significant seasonal oscillation in the strength of the vertical shear of the current. Qiu estimates a seasonal variation of the order of $50 \%$ of the mean value of the shear (from a minimum of $4 \mathrm{~cm} \mathrm{~s}^{-1}$ to a maximum of $6 \mathrm{~cm} \mathrm{~s}^{-1}$ ). In order to deal with the variability of the currents a set of stability calculations using steady theory based on several 'snapshot' profiles of the currents in various seasons was performed by Qiu to explain the strong seasonal variability of the observed eddy kinetic energy. However, such calculations of the observed 'snapshot' flow field yielded growth rates for the instability ranging from 60 to 180 days, clearly of the same order as, if not longer than, the time scale over which the currents change significantly. Certainly, a model of the current as steady would seem to be problematic. While our idealizations do not allow a direct comparison with observations, we suggest that the mechanism investigated here is applicable to such situations.

\section{Formulation of the linear problem}

We consider the stability of the zonal flow in the standard quasi-geostrophic two-layer model on the beta-plane (see for example Pedlosky 1987). The governing equations for the nonlinear evolution of the disturbances are, in non-dimensional form,

$$
\left[\frac{\partial}{\partial t}+U_{n} \frac{\partial}{\partial x}\right] q_{n}+\frac{\mathrm{d} Q_{n}}{\mathrm{~d} y} \frac{\partial \phi_{n}}{\partial x}+J\left(\phi_{n}, q_{n}\right)=-\mu q_{n}, \quad n=1,2
$$

Here the subscript $n$ refers to the upper $(n=1)$ and lower $(n=2)$ layers. The disturbance streamfunction is $\phi_{n}(x, y, t)$ in each layer. The perturbation potential 
vorticity in each layer is defined as

$$
\begin{aligned}
& q_{n}=\nabla^{2} \phi_{n}+F(-1)^{n}\left[\phi_{1}-\phi_{2}\right], \\
& F=\frac{f^{2} L^{2}}{g^{\prime} D}, \quad \beta=\frac{2 \Omega \cos \theta}{R} \frac{L^{2}}{U} .
\end{aligned}
$$

The variables in the definition for $F$ are standard: $f$ is the Coriolis parameter, $L$ is the width of the channel, $g^{\prime}$ is the reduced gravity. For algebraic simplicity the undisturbed layer thickness is $D$ for each layer. Note that now $0 \leqslant y \leqslant 1$. The nondimensional parameter $\beta$ measures the effect of the Earth's sphericity. $\Omega$ is the Earth's rotation rate, $R$ its radius and $\theta$ is the central latitude of the tangent beta-plane to the Earth's surface. Horizontal velocities are scaled with a characteristic speed of the mean flow, $U$, and time is scaled with the advective time $L / U$.

The potential vorticity gradient of the mean flow is in these non-dimensional units

$$
\frac{\mathrm{d} Q_{n}}{\mathrm{~d} y}=\beta-F(-1)^{n}\left(U_{1}-U_{2}\right) .
$$

The zonal velocities in each layer $U_{n}$ are functions of time. The nonlinear advection of perturbation potential vorticity is given by the Jacobian of $\phi_{n}$ and $q_{n}$ with respect to $x$ and $y$. For the linear problem this term is ignored. The dissipation is represented as a simple decay law for potential vorticity with coefficient $\mu$. This form is chosen purposely for, as has been shown (Klein \& Pedlosky 1992), such a form for the dissipation for the case of steady shears will force the final, finite-amplitude wave state to equilibrate to a steady state rather than yielding either self-maintained periodic motions or chaotic behaviour. In this way we can identify periodic or chaotic behaviour in the present problem as being due to the presence of the oscillating basic shear.

We first consider the linear problem. Ignoring the Jacobian we consider solutions of the form

$$
\phi_{n}=A_{n}(t) \mathrm{e}^{\mathrm{i} k x} \sin \left(m_{p} y\right)+*,
$$

where $m_{p}$ is any integral multiple of $\pi$ and where an asterisk denotes the complex conjugate of the preceding expression. We obtain two coupled ordinary differential equations in time for the wave amplitudes:

$$
\begin{gathered}
\left(\frac{\mathrm{d}}{\mathrm{d} t}+\mathrm{i} k U_{1}\right)\left[-K^{2} A_{1}+F\left(A_{2}-A_{1}\right)\right]+\frac{\mathrm{d} Q_{1}}{\mathrm{~d} y} \mathrm{i} k A_{1}=0, \\
\left(\frac{\mathrm{d}}{\mathrm{d} t}+\mathrm{i} k U_{2}\right)\left[-K^{2} A_{2}+F\left(A_{1}-A_{2}\right)\right]+\frac{\mathrm{d} Q_{2}}{\mathrm{~d} y} \mathrm{i} k A_{2}=0, \\
K^{2}=k^{2}+\left(m_{p}\right)^{2} .
\end{gathered}
$$

We have apparently ignored the presence of dissipation. However, in the linear problem it is easily restored in the final result by simply subtracting $\mu$ from the growth rate to be obtained without dissipation. We recall that the mean flow is a function of time and, to this point, arbitrary. It is also convenient to recast the problem in terms of the barotropic and baroclinic streamfunction amplitudes, i.e. writing

$$
\begin{gathered}
A_{B}=A_{1}+A_{2}, \quad A_{T}=A_{1}-A_{2}, \\
U_{m}=\left(U_{1}+U_{2}\right) / 2, \quad U_{s}=\left(U_{1}-U_{2}\right) / 2,
\end{gathered}
$$


we obtain

$$
\begin{gathered}
\frac{\mathrm{d} A_{B}}{\mathrm{~d} t}+\mathrm{i} k\left[U_{m}-\beta / K^{2}\right] A_{B}=-\mathrm{i} k U_{s} A_{T}, \\
\frac{\mathrm{d} A_{T}}{\mathrm{~d} t}+\mathrm{i} k\left[U_{m}-\beta /\left(K^{2}+F\right)\right] A_{T}=-\mathrm{i} k U_{s} \frac{K^{2}-2 F}{K^{2}+2 F} A_{B} .
\end{gathered}
$$

Consider the situation in which $U_{m}$ is independent of time and only the shear varies (this is a restriction easily relaxed with no loss of generality). We rewrite the problem's variables as

$$
\begin{gathered}
A_{B}=B_{B} \exp \left(-\mathrm{i} k t\left(U_{m}-\left[c_{b}+c_{t}\right] / 2\right)\right), \quad A_{T}=B_{T} \exp \left(-\mathrm{i} k t\left(U_{m}-\left[c_{b}+c_{t}\right] / 2\right)\right), \\
c_{b}=\beta / K^{2}, \quad c_{t}=\beta /\left(K^{2}+F\right), \\
U_{s}=U_{s 0}(1+\delta(\vartheta)), \\
K^{2}=a^{2} F, \quad b=\beta / F U_{s 0}, \quad \vartheta=k U_{s 0} t .
\end{gathered}
$$

The last relation, $(2.8 h)$, implies that we have scaled time with the advective time scale. We obtain the ordinary differential equations for the amplitudes $B_{B}$ and $B_{T}$ :

$$
\begin{gathered}
\frac{\mathrm{d} B_{B}}{\mathrm{~d} \vartheta}-\mathrm{i} \frac{b}{a^{2}\left(a^{2}+2\right)} B_{B}=-\mathrm{i}(1+\delta) B_{T}, \\
\frac{\mathrm{d} B_{T}}{\mathrm{~d} \vartheta}+\mathrm{i} \frac{b}{a^{2}\left(a^{2}+2\right)} B_{T}=-\mathrm{i}(1+\delta) \frac{\left(a^{2}-2\right)}{\left(a^{2}+2\right)} B_{B} .
\end{gathered}
$$

The quantity $\delta(\vartheta)$ represents the increment of the shear above a critical value. For example, for each wavenumber $a$ the problem with the steady shear, i.e. $\delta=0$, possesses a critical value of shear given by

$$
b=\left[a^{4}\left(4-a^{4}\right)\right]^{1 / 2} .
$$

If we were to consider the nature of only the linear problem we could deal directly with equations (2.9), However, since we will subsequently consider the finite-amplitude behaviour of the waves we will, in this study, restrict our attention to the vicinity of the marginal curve. The nature of the resulting linear problem is qualitatively identical to the system $(2.9 a, b)$. Note that for instability of the classical (steady) type $a^{2}<2$.

We thus choose $b$ to be given by (2.10) so that we are examining the role of the oscillating shear in the neighbourhood of the classical marginal curve for instability. For small $\delta$, i.e. near the marginal curve, a perturbation expansion is useful. We let $\Delta$ be a small parameter which measures the distance from the critical shear and

$$
\delta=\Delta G+\Delta^{1 / 2} H \cos \omega \vartheta
$$

where $G=O(1), H=O(1)$.

Thus $\Delta G$ represents the small, additional steady increment of the shear above critical and $\Delta^{1 / 2} H$ represents a larger value of the amplitude of the oscillating part of the shear. We also introduce a slow time variable $T=\Delta^{1 / 2} \vartheta$ and allow the amplitudes to be functions of both $\vartheta$ and $T$. An expansion of the form

$$
B_{B, T}=B_{B, T}^{(0)}+\Delta^{1 / 2} B_{B, T}^{(1)}+\Delta B_{B, T}^{(2)}+\cdots
$$

easily yields the following results:

at lowest order,

$$
B_{B}^{(0)}=B_{B}(T), \quad B_{T}^{(0)}=r B_{B}^{(0)}
$$


where

and at $O\left(\Delta^{1 / 2}\right)$

$$
r^{2}=\left[\frac{2-a^{2}}{2+a^{2}}\right]
$$

$$
\begin{array}{r}
B_{B}^{(1)}=\frac{-H}{\omega^{2}}\left(2 r^{2} \cos \omega \vartheta+\mathrm{i} r \omega \sin \omega \vartheta\right) B_{B}^{(0)}, \\
B_{T}^{(1)}=\mathrm{i} \frac{\partial B_{B}^{(0)}}{\partial T}+\frac{\mathrm{i} H}{\omega^{2}}\left(r^{2} \omega \sin \omega \vartheta+2 \mathrm{i} r^{3} \cos \omega \vartheta\right) B_{B}^{(0)},
\end{array}
$$

while removing secular terms at order $\Delta$ yields the evolution equation,

$$
\frac{\partial^{2} B_{B}^{(0)}}{\partial T^{2}}+B_{B}^{(0)}\left\lfloor-2 G r^{2}+\frac{H^{2} 2 r^{2}}{\omega^{2}}\right\rfloor=0 .
$$

Thus, while an increment of the steady shear above critical, $G$, would give rise to exponentially growing disturbances it is evident from (2.15) that an oscillating shear will stabilize the flow, a balance occurring when $G=H^{2} / \omega^{2}$. This stabilization of the unstable shear flow by an oscillating component is analogous to the well-known stabilization of the unstable equilibrium of an up-ended pendulum by a periodic oscillation of the point of support of the pendulum.

Similarly, the oscillation can destabilize a subcritical shear if the frequency is appropriately tuned. As mentioned above, one can find that phenomenon with the equation set $(2.9 a, b)$ but as is apparent from (2.15) the effect of the oscillating shear will be more powerful at lower frequencies. In the treatment given above, a relatively large $O\left(\Delta^{1 / 2}\right)$ value of the oscillating shear is needed. However, if the frequency is of the order of the growth rate, i.e. $O\left(\Delta^{1 / 2}\right)$ instead of the advective time as in the previous treatment the linear problem actually simplifies while enhancing the role of the oscillating shear.

If one returns to (2.9), (2.10) and (2.11) and rescales the frequency such that

$$
\begin{gathered}
\omega \rightarrow \Delta^{1 / 2} \omega \\
\delta(T)=\Delta(G+H \cos \omega T)
\end{gathered}
$$

a development analogous to (2.12) et seq. gives rise to the amplitude equation,

$$
\frac{\partial^{2} B_{B}^{(0)}}{\partial T^{2}}-2 r^{2}[G+H \cos \omega T] B_{B}^{(0)}=0 .
$$

This is the classical Mathieu equation (see, for example, Morse \& Feshbach 1953 or Cole 1968).

Figure $1(a)$ shows the stabilization process described above as given by (2.17). Here $G=0.01, H=0.52, \omega=2$, and $\mu=0.001$. Although the time-averaged shear is supercritical the time-varying shear stabilizes the perturbation.

In the current notation destabilization occurs at a series of critical frequencies for the case when the mean shear is stable, i.e. when $G<0$. In the limit $H \rightarrow 0$ these frequencies are given as follows. For slightly subcritical shears a natural frequency of oscillation of the system is given by

$$
\sigma \equiv\left[-2 r^{2} G\right]^{1 / 2}
$$

and the critical frequencies for so-called parametric instability occur when

$$
\omega=\frac{2}{n} \sigma, \quad n=1,2, \ldots
$$



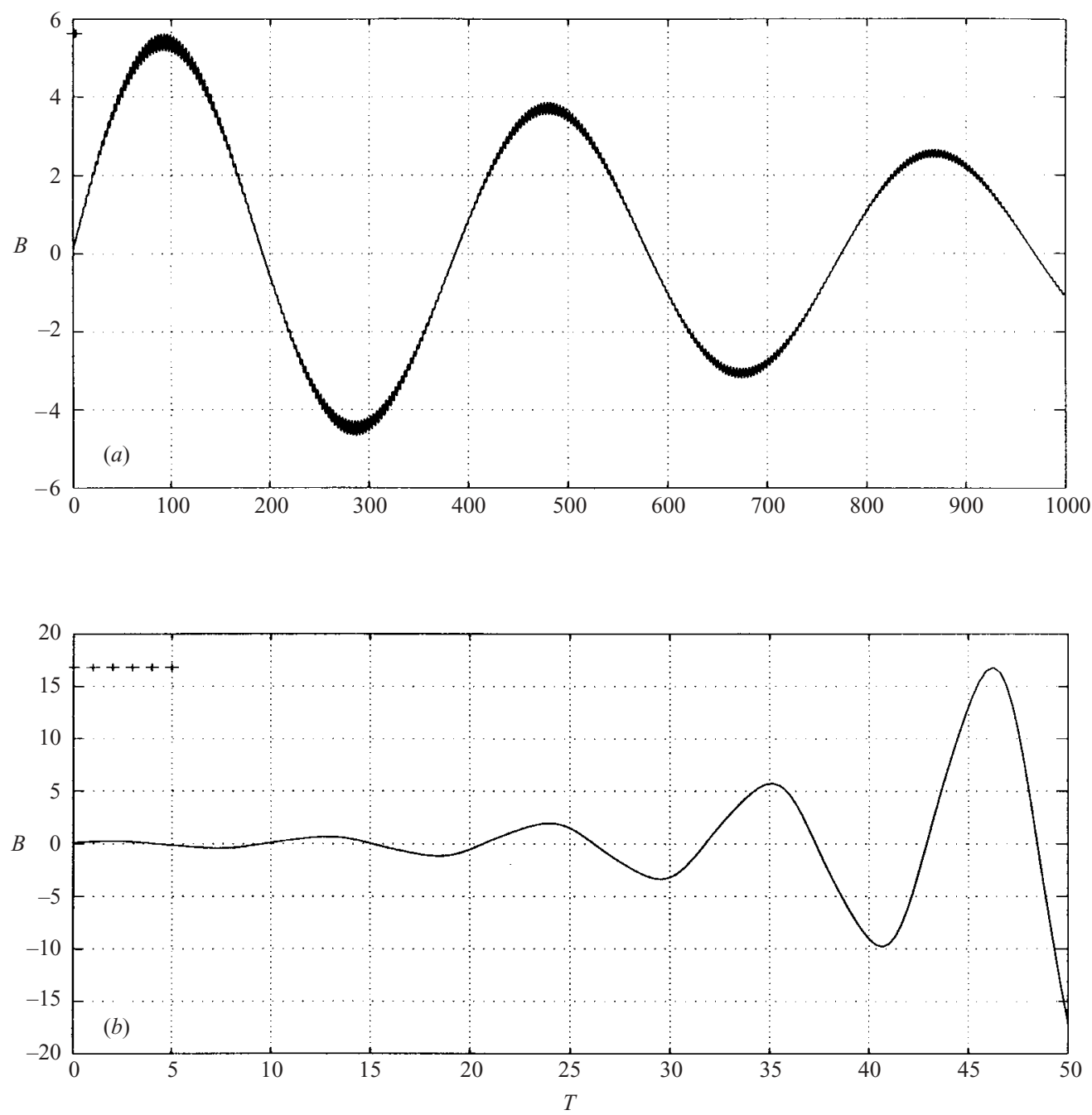

FIGURE 1. The amplitude behaviour in the linear system (2.17) for the case when $a=(2.1)^{1 / 4}$. (a) The stabilization of a steady unstable shear by an oscillating component, $G=0.01$, $H=0.52, \mu=0.001, \omega=2$. (b) The parametric destabilization of a steady shears flow, $G=-1$, $H=0.7$, and $\omega=1.1 .303$, the first critical Mathieu frequency. Note the exponentially increasing amplitude although the time-mean shear is subcritical. The line of cross-hatches in the figure describes the period of the imposed oscillatory shear.

It is important to note that as $H$ increases, the range of $\omega$ for which instability occurs expands until for large enough $H$ the system is unstable for most frequencies. The reader is referred to Morse \& Feshbach (1953) for a complete discussion of this linear system. The perturbations with the largest growth rates correspond to the $n=1$ mode corresponding to a frequency which, for small $H$, is twice the natural frequency $\sigma$. Note, that since $\sigma$ depends on the closeness of the shear to the marginal curve the time scales involved can be very long.

Figure 1(b) shows the amplitude behaviour for the linear system when we have chosen $a$ to be slightly greater than the wavenumber of minimum critical shear for the classical problem $\left(a=(2.1)^{1 / 4}\right)$ for $G=-1$ and $H=0.7$. Thus, the 
time-averaged shear is stable and, indeed, the instantaneous value of the shear is always less that the critical value of the steady-state problem. Nevertheless, due to the parametric instability the disturbance will exponentially grow while oscillating at the frequency $\sigma$.

The destabilization of hydrodynamic systems by parametric instability when the background state is time dependent is not a new phenomenon. For the case of shear flows von Kerczek (1982) showed both the parametric stabilization and destabilization of plane Poiseuille flow. Rosenblat (1968) examined the parametric instability of the Taylor centrifugal instability, again showing the Mathieu-like properties of the linear fluid system. More recently Poulin, Flierl \& Pedlosky (2003) investigated the parametric instability of a barotropic shear flow, concentrating on the destabilization of the linear problem. Similarly, Majda \& Shefter (1998) have examined the instability of a class of simple but cleverly chosen stratified non-rotating flows whose parametric instabilities can be examined within a linear theory even for finite amplitude. In fact, the linear problem can be extended in several interesting directions. From the point of view of this study, perhaps the most interesting result in the mathematical literature on the Mathieu equation concerns mean flows whose time dependence is not periodic. It is possible to show (Davis \& Rosenblat 1980; Zounes \& Rand 1998) that if the function $\delta(T)$ is made up of a series of periodic components each with frequency $\omega_{j}$ the analysis given above follows whenever $\sigma$ is close to each one of the infinite set given by (2.19) for each separate $\omega_{j}$. That is, each oscillatory component of the basic shear flow can be treated independently when the natural frequency coincides with one of the Mathieu resonance frequencies. Of course, if $\delta(T)$ is composed of a series of such terms of the form

$$
\delta(T)=\Delta\left(G+\sum_{j} H_{j} \cos \omega_{j} T\right),
$$

the amplitude of each will be diminished if the overall variance amplitude of the oscillation is fixed. Since the growth rate of the most dangerous instability (corresponding to $n=1$ in (2.19)) will scale with $H_{j}$, the parametric instability will weaken as more frequencies are added. On the other hand, the set $\omega_{j}$ need not correspond to frequencies which are rational multiples of each other so that there is no necessity to restrict attention to shear flows that are strictly periodic in time. In all our calculations and discussion of the nonlinear problem we will, for simplicity, restrict attention to a single periodic component but it is important to realize that this is much less restrictive an assumption than it first appears.

When the frequency is small a WKB solution to (2.17) is possible. Using standard methods one obtains the asymptotic solution for small $\omega$ but $G$ and $H$ of $O(1)$ in the form

$$
B_{0}=\frac{A}{\delta^{1 / 4}} \exp \left(\sqrt{2 r} \int_{0}^{t} \delta^{1 / 2} \mathrm{~d} t^{\prime}\right) .
$$

Positive $\delta$ contributes to growth but negative $\delta$ instead leads to an oscillation and, consistent with (2.15), large enough $H$ can stabilize the linear problem. However, if the period of time for which $\delta$ is positive is large enough the amplitude will grow sufficiently to invalidate linear theory. It seems rather artificial to talk about ultimate linear instability or stability if the amplitude has already exceeded the limits of linear theory for some interval of time. Therefore, rather than extend the linear problem we have found it of greater interest to examine the nonlinear extension of the problem. 


\section{The nonlinear problem}

The weakly nonlinear theory is developed in the vicinity of the marginal curve of the classical steady shear problem. That curve relates the steady shear $b$ and the wavenumber $a$ and is given by (2.10). The perturbation method required to achieve an amplitude equation governing the nonlinear evolution of the wave is standard and can be found described in many places, e.g. Pedlosky (1987) so that only the briefest outline of the development is given here. Naturally, a full understanding of the nonlinear problem must eventually move beyond weakly nonlinear theory but it seems to us useful to start with a dynamics in which the novel character of the timedependent problem is more easily clarified as in the weakly nonlinear perturbation problem.

Returning to (2.1) we again write the shear of the basic state in the form

$$
U_{s}=U_{s 0}(1+\delta(t)), \quad \delta=\Delta(G+H \cos (\varepsilon \alpha \omega t)) ;
$$

$\alpha$ is an order-one scaling constant that we will choose below and $\Delta$ is a measure of the super- (or sub-) criticality of the flow, $\varepsilon=O\left(\Delta^{1 / 2}\right)$ so that the frequency $\omega$, which is order 1 , represents a slow oscillation of the basic shear. We have related $\Delta$ and $\varepsilon$ by the scaling choice

$$
\varepsilon=O\left(\Delta^{1 / 2}\right)
$$

so as to balance linear growth and nonlinear stabilization. The dissipation is also chosen so that $\mu=O(\varepsilon)$. This sets, a priori, the growth rate, the oscillation period and the dissipation time scale to be of the same order although subsequently we will allow numerical differences.

The perturbation streamfunctions for the barotropic and baroclinic components of the flow are each expanded as

$$
\begin{aligned}
\phi_{B} & =\varepsilon\left[\phi_{B}^{(0)}+\varepsilon \phi_{B}^{(1)}+\varepsilon^{2} \phi_{B}^{(2)}+\cdots\right], \\
\phi_{T} & =\varepsilon\left[\phi_{T}^{(0)}+\varepsilon \phi_{T}^{(1)}+\varepsilon^{2} \phi_{T}^{(2)}+\cdots\right] .
\end{aligned}
$$

We assume that each field is a function of both $t$, the advective scale, and $T$, the relatively slow growth rate time scale, where $T=\varepsilon t$. With $\varepsilon \ll 1$, insertion of the series (3.3) into (2.1) along with the scaling relations among $\Delta, \mu$ and $\varepsilon$, leads to the following results.

At lowest order the fields are given by

$$
\begin{aligned}
& \phi_{B}^{(0)}=\frac{A_{B}(T)}{2} \mathrm{e}^{\mathrm{i} k(x-c t)} \sin \left(m_{p} y\right)+*, \\
& \phi_{T}^{(0)}=\frac{A_{T}(T)}{2} \mathrm{e}^{\mathrm{i} k(x-c t)} \sin \left(m_{p} y\right)+*,
\end{aligned}
$$

where an asterisk represents complex conjugation of the previous term. At the marginal curve, with (2.8),

$$
c=\left(c_{b}+c_{t}\right) / 2
$$

and where without loss of generality we have taken the mean flow of the two layers, $U_{m}$, to be zero. We are examining the finite-amplitude dynamics of a single wavelike perturbation and in principle, as shown by Pedlosky (1982), one needs to avoid the critical layer behaviour which occurs at $a^{2}=2^{1 / 2}$, i.e. the maximum of (2.10). That complication can easily be avoided by restricting attention to a narrow enough channel so that $a$ always exceeds that value. 
Furthermore, at the marginal curve,

$$
A_{T}=\left(\frac{c_{t}-c_{b}}{2 U_{s 0}}\right) A_{B}
$$

At the next order in $\varepsilon$ we obtain

$$
\begin{gathered}
\phi_{B}^{(1)}=0, \\
\phi_{T}^{(1)}=\frac{\mathrm{i}}{2 k U_{s 0}}\left(\frac{\partial}{\partial T}+\frac{\mu}{\varepsilon}\right) A_{B} \mathrm{e}^{\mathrm{i} k(x-c t)} \sin \left(m_{p} y\right)+*+\Phi_{T}(y, T),
\end{gathered}
$$

where the function $\Phi_{T}(y, T)$ represents a nonlinear $\left(O\left(\varepsilon^{2}\right)\right)$ correction to the mean shear. Continuing further to the next order in $\varepsilon$ involves some algebra but a straightforward calculation in which secular terms are removed to render the problem uniformly valid to this order leads to two coupled equations for the unknowns $\Phi_{T}(y, T)$ and $A_{B}$ :

$$
\begin{aligned}
& \left(\frac{\partial}{\partial T}+\frac{\mu}{\varepsilon}\right)^{2} A_{B}-2 k^{2} U_{s 0}^{2} \frac{2 F-K^{2}}{2 F+K^{2}} \frac{\delta}{\varepsilon^{2}} A_{B} \\
& \quad+k^{2} U_{s 0} \frac{4 m_{p}\left(K^{2}-F\right) A_{B}}{K^{2}\left(2 F+K^{2}\right)} \int_{0}^{1}\left\{\frac{\partial^{2} \Phi_{T}}{\partial y^{2}}-\Phi_{T} \frac{\left(2 F-K^{2}\right) K^{2}}{\left(K^{2}-F\right)}\right\} \mathrm{d} y=0 \\
& \left(\frac{\partial}{\partial T}+\frac{\mu}{\varepsilon}\right)\left\{\frac{\partial^{2}}{\partial y^{2}} \Phi_{T}-2 F \Phi_{T}\right\}=\left.\frac{m_{p} F}{2 U_{s 0}}\left[\frac{\partial}{\partial T}+\frac{2 \mu}{\varepsilon}\right] A_{B}^{(0)}\right|^{2} \sin 2 m_{p} y
\end{aligned}
$$

Solutions of $(3.8 b)$ can be found by first solving

$$
\frac{\partial^{2} \Phi_{T}}{\partial y^{2}}-2 F \Phi_{T}=\frac{m_{p} F}{2 U_{s 0}}\left(|A|^{2}+\hat{P}(T)\right) \sin 2 m_{p} y,
$$

where for notational simplicity we have defined $A_{B}=A$ and where $\hat{P}(T)$ is a function of $T$ that must satisfy

$$
\left(\frac{\partial}{\partial T}+\frac{\mu}{\varepsilon}\right) \hat{P}=\frac{\mu}{\varepsilon}|A|^{2} .
$$

The equation for the wave amplitude then becomes

$$
\left\lfloor\frac{\mathrm{d}^{2} A}{\mathrm{~d} T^{2}}+2 \frac{\mu}{\varepsilon} \frac{\mathrm{d} A}{\mathrm{~d} T}+\left(\frac{\mu}{\varepsilon}\right)^{2} A\right\rfloor-2 k^{2} U_{s 0}^{2} r^{2} \frac{\delta}{\varepsilon^{2}} A+A N\left[|A|^{2}+\hat{P}\right]=0,
$$

where $N$ is a complicated algebraic expression given in the Appendix. A minor rescaling will put the above equations in a form without most parameters. Writing

$$
\begin{gathered}
\tau=\sigma_{0} T, \quad \sigma_{0}=\left(\frac{K U_{s 0} \Delta^{1 / 2}}{\varepsilon}\right), \\
A=\frac{K U_{s 0} \Delta^{1 / 2}}{\varepsilon N^{1 / 2}} B, \quad \hat{P}+A^{2}=\frac{K^{2} U_{s 0}^{2} \Delta}{\varepsilon^{2} N} P,
\end{gathered}
$$


leads to the final set of amplitude equations,

$$
\begin{gathered}
\left(\frac{\mathrm{d}}{\mathrm{d} \tau}+\mu\right)^{2} B-2 r^{2} B(G+H \cos (\omega \tau)) B+B P=0, \\
\left(\frac{\mathrm{d}}{\mathrm{d} \tau}+\mu\right) P=2 B\left(\frac{\mathrm{d} B}{\mathrm{~d} \tau}+\mu B\right),
\end{gathered}
$$

where $\alpha$ has been chosen to be the ratio between $T$ and $\tau$, i.e. $\sigma_{0}$ and $\mu$ has been redefined,

$$
\frac{\mu}{\varepsilon \sigma_{0}} \rightarrow \mu \text {. }
$$

To recapitulate, the solution of (3.13) for $B(T)$ yields, with (3.7) and (3.12), the amplitude of the barotropic component of the unstable wave field. The baroclinic component, to lowest order, is then obtained from (3.6). The amplitude of the correction to the mean flow follows from the solution $P(T)$ and the spatial structure is given by the solution to (3.9). Thus, the system (3.13) will be investigated in more detail in the following sections. If $H=0$, that is if the shear of the basic state is independent of time, the system (3.13) can be put in the form of the Lorenz equations (Klein \& Pedlosky 1992). A detailed comparison of the two systems shows, however, that this set is equivalent to the Lorenz equations for a parameter setting for the latter in which the Prandtl number for the Lorenz equations is equal to unity. For such a Prandtl number in the Lorenz equations the solutions always reach a steady state in finite amplitude. No chaotic or self-maintained periodic behaviour can take place. This is an important consideration for it implies that if we find periodic or aperiodic solutions with non-trivial time dependence it is due to the presence of the time-varying shear, i.e. $H$ different from zero. Figure 2 shows such an equilibration occurring for the steady flow case, i.e. for $H=0$. The solution for $B(T)$ swiftly equilibrates to the equilibrium value predicted by (3.13). In the next sections we take up the behaviour of the system (3.13) when the shear is time dependent, i.e. when $H \neq 0$.

\section{Finite-amplitude solutions $G>0$}

Since the time scale has been chosen to correspond to the linear growth rate for slight supercriticality the only significant values for $G$ are either -1 (subcritical states), 0 (precisely marginal states) or +1 (supercritical states). In this section we describe the role of time-dependent shear when the flow is supercritical, i.e. $G=1$. The behaviour of the solutions is a complex function of the parameters $H, \mu$ and $\omega$ and it is beyond the scope of this paper to give more than an indication of that dependence.

Figure 3 shows an overall regime diagram in the $(H, \omega)$-plane constructed for the case $\mu=0.01$ and $G=1$. Three qualitatively different behaviours are noted in the figure. Smooth periodic solutions with the period of the shear's oscillation are denoted with circles, essentially periodic but ragged oscillations are denoted by squares and aperiodic solutions with broad spectra with no sharp peaks are denoted by asterisks. Figure 4 shows the solution, $B(T)$, for relatively small $H=0.1$, small enough so that the flow is at all times above the critical shear. The disturbance amplitude has settled into a finite-amplitude limit cycle at the frequency of the shear's oscillation. The figure shows the solution after it has settled into a periodic oscillation. Recall that the non-dimensional time used in the figure is the dimensional time scaled with the characteristic e-folding time $L / U \Delta^{-1 / 2}$. 


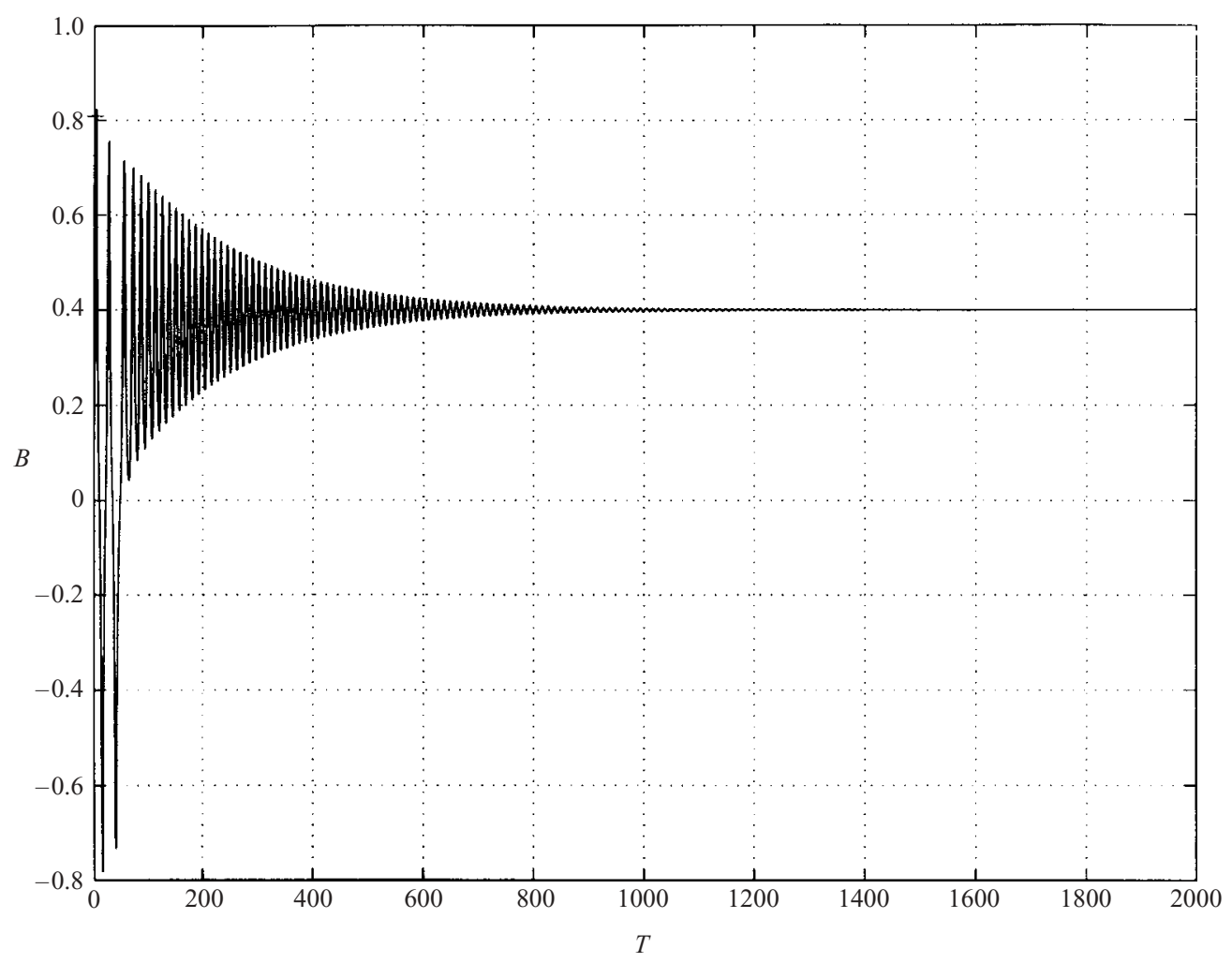

FIGURE 2. The amplitude evolution for the case of slightly supercritical but steady shear, $G=1, H=0, \mu=0.01$. The solution equilibrates to a steady finite-amplitude equilibrium.

As is evident by eye, the solution is dominated by a single frequency with minor contributions, many orders of magnitude smaller, of higher harmonics of this fundamental. This is verified by a spectral analysis performed on the time series of the calculation but the details are not shown here.

For $H>1$ the solution background flow experiences periods of subcriticality even though, on average, the flow is supercritical. For some values of $H>1$ the solution, while remaining periodic, becomes rather intricate containing a rich frequency content. Figure 5 shows such an oscillation (for $H=1.5$ ) and its rather lovely phase plane portrait, which emphasizes the complexity of the solution while remaining periodic. Finally, for an intermediate value of $H$, essentially aperiodic chaotic behaviour is obtained. Figure 6 shows the solution at a smaller value of $H=0.4$ and a smaller damping rate $(\mu=0.001)$ which clearly shows aperiodic behaviour. Such behaviour is also found for larger dissipation $(\mu=0.01)$.

A wide array of behaviour is possible, especially when $H$ is large enough to render the flow stable for some period of time and the dissipation is small enough. This is especially true if $\omega$ is small. Then the solution grows rapidly during periods of positive supercriticality only to decay when the supercriticality is negative. For small $\mu$ some disturbance is maintained until the next season of growth but the slightly different value of the amplitude occurring at the initiation of the new period of growth renders the solution aperiodic. Figure 7 shows such a case when $G=1, H=1.5 \mu=0.001$, and $\omega=0.01$. This, in comparison with the solution of figure 5 , demonstrates a typical 


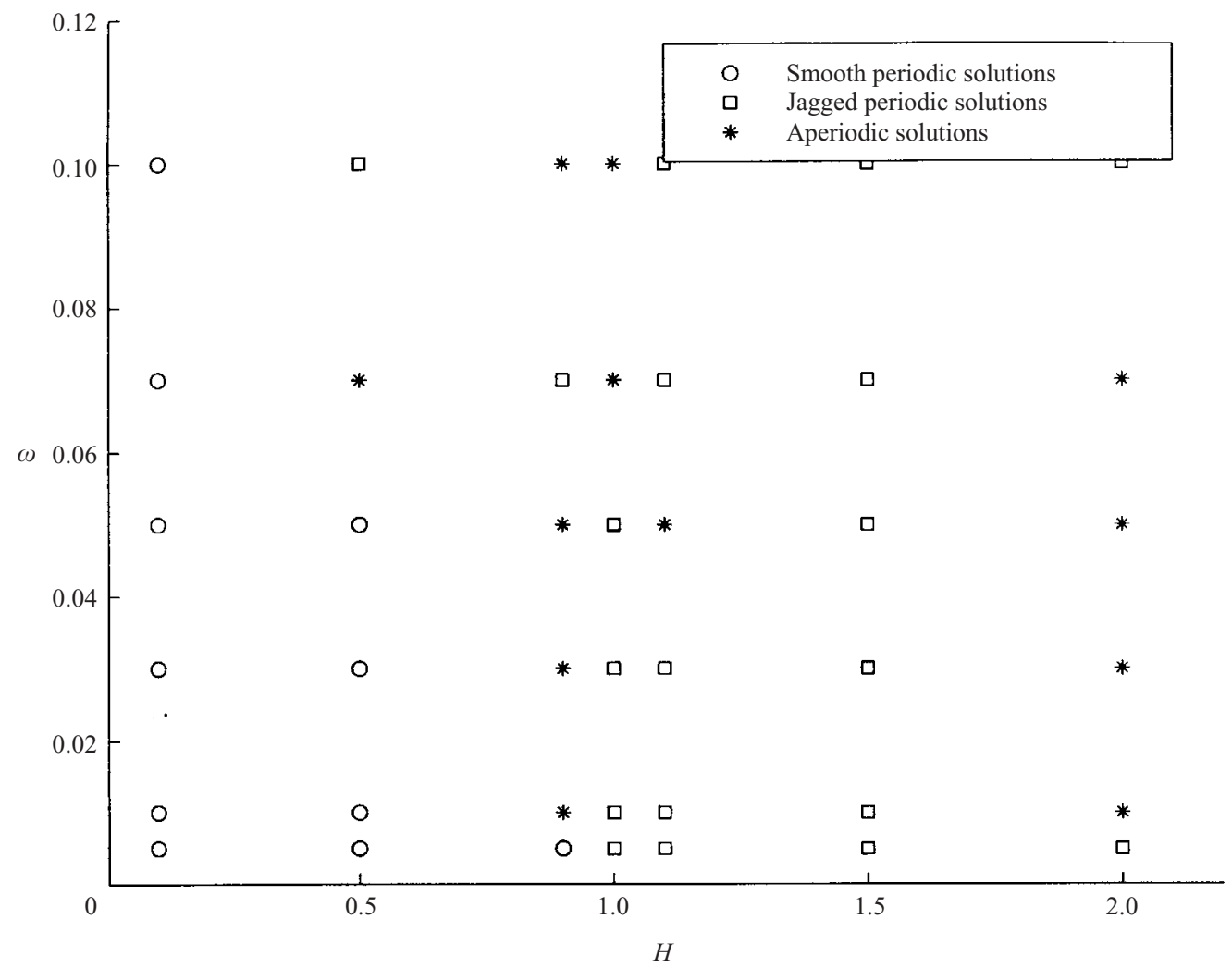

FIGURE 3. The regime diagram in the $(H, \omega)$-plane constructed for the case $\mu=0.01$ and $G=1$. Three qualitatively different behaviours are noted in the figure. Smooth periodic solutions with the period of the shear's oscillation are denoted with circles, essentially periodic but ragged oscillations are denoted by squares, and aperiodic solutions with broad spectra with no sharp peaks are denoted by asterisks.

dependence on dissipation. There is increased aperiodic behaviour found for smaller dissipation.

Figure $7(a)$ shows the total time history in which the aperiodic nature of the solution is evident. Figure $7(b)$ shows the detail of the last $5 \%$ of the time history. In this figure the supercriticality is shown with a dashed line superimposed on the solution. It is clear from the figure that the amplitude grows rapidly during periods of positive supercriticality and then is almost quenched during periods of subcriticality. However, the nonlinear oscillation occurring in the period of subcritical shear forms the initial state for the subsequent 'season' of growth. Since the nonlinear oscillation (of the nonlinear inviscid type discussed in Pedlosky 1987) during the interval of subcriticality has a period incommensurate with the period of the background shear, the initiation of growth always starts with different values of the amplitude leading to an overall aperiodicity in behaviour.

\section{Finite-amplitude solutions $G<0, H>1$}

When $G<0$ the time-averaged shear is always subcritical. A large enough value of $H$ will raise the shear temporarily above the classical threshold. The behaviour of the 


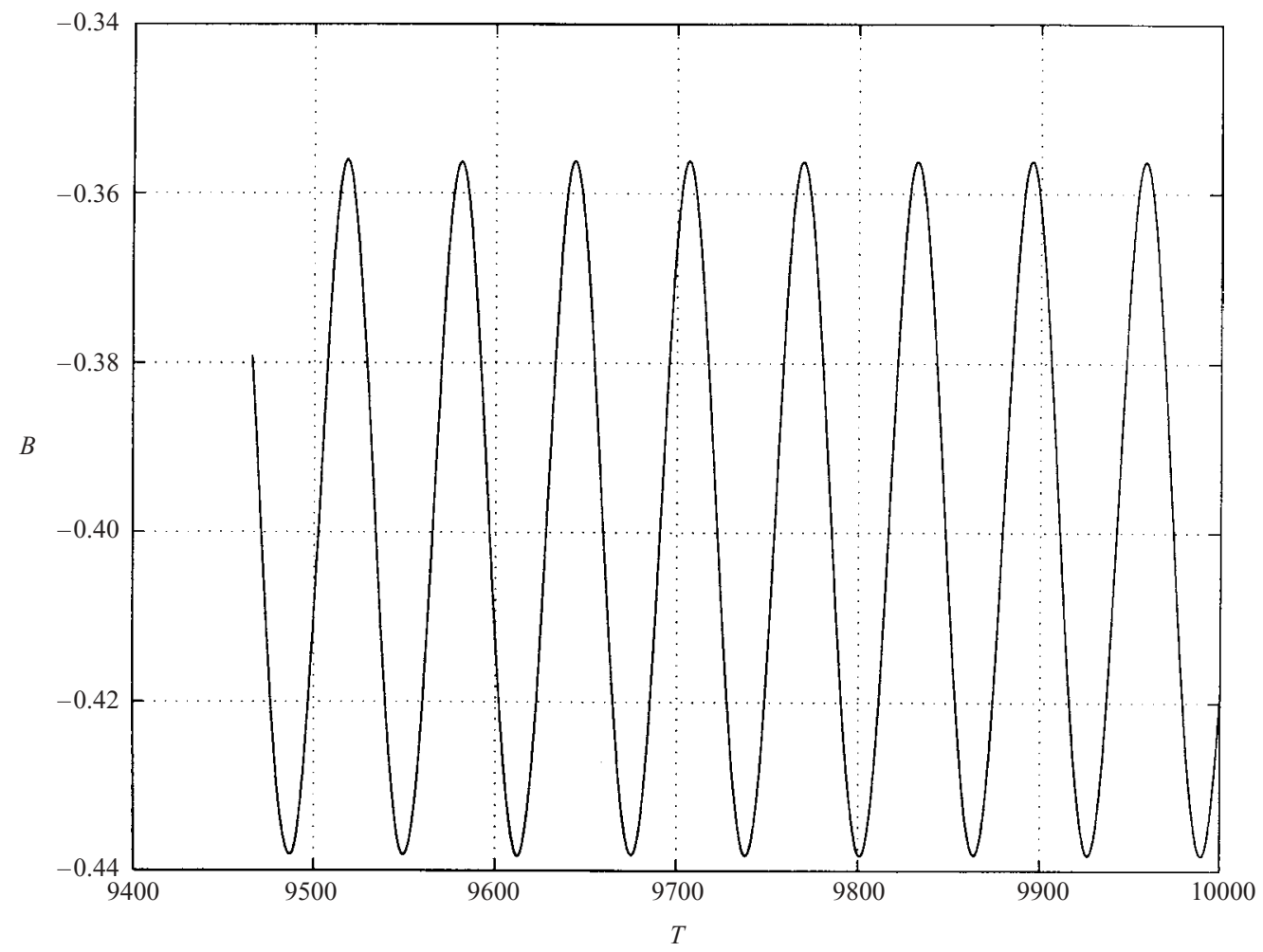

FIGURE 4. The finite-amplitude periodic solution for $G=1, H=0.1, \omega=0.1, \mu=0.01$. The solution is shown for the last 600 time units of a run of length $T=10000$. The solution has settled into a limit cycle with a period equal to the period of the oscillating shear.

system when $H>1$ is discussed in this section. Values of $H<1$ are discussed in the following section.

Figure 8 is a rough regime diagram for $G=-1$ in the $(H, \omega)$-plane. For small $\omega$ and $H<1$ most solutions, except for regions described in $\S 6$, eventually decay to zero. There is an abrupt transition at $H=1$. For $H \geqslant 1$ the shear flow is, for some time intervals, above the classical curve denoting instability. For $H$ only slightly greater than 1 this results in complex periodic solutions of the kind shown in figure 9 where $H=1.1$. Again the intervals of positive and negative instantaneous values of supercriticality are given by the dashed curve. The intervals of slight instability (where the dashed curve is above the zero line) correspond to the intervals of maximum disturbance amplitude. Again, the result is easily understood as a seasonal interval of instability occurring slowly enough to allow the perturbation to respond in a classical way to the opportunity for baroclinic instability. What is surprising is the nearly precise periodicity of the solution as demonstrated by the phase-plane portrait in figure $9(b)$. On the other hand, larger values of $H$ generally lead to aperiodic behaviour (although as we see in figure 8 there is a complex frequency dependence on this transition). Figure 10 shows a calculation for the same values of $\omega, \mu$ and $G$ but with a value of $H=2.2$. Again, the strong 'seasonal' transition between intervals of instability and stability for $H>1$ renders the solution aperiodic and to the eye, chaotic. The spectral analysis of the solution, although not shown here, clearly demonstrates a broad power density spectrum with no sharp peaks. There is a 

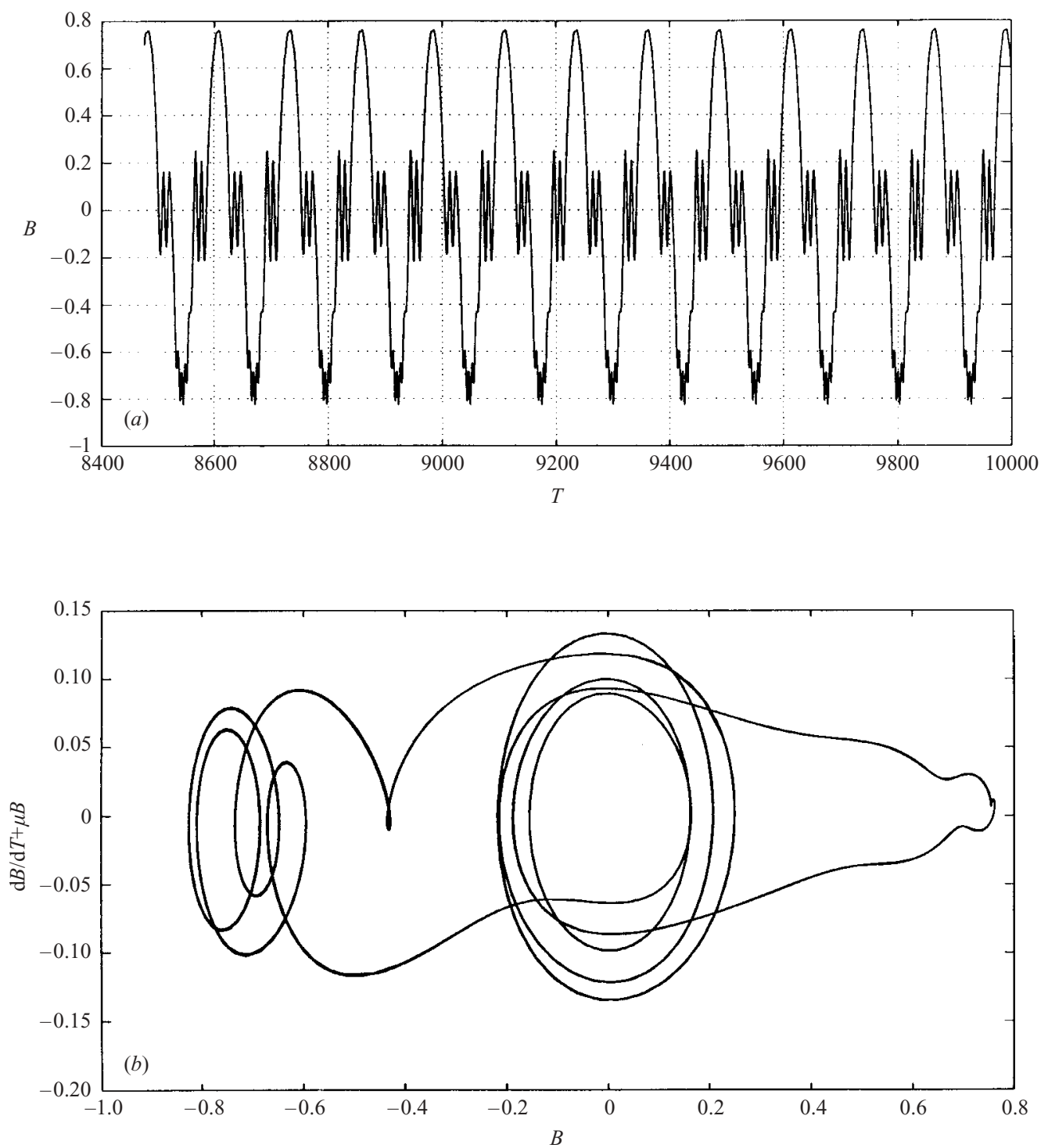

FIGURE 5. As in figure 4 except that $H=1.5$. (a) The time history of the solution.

(b) The phase plane.

broad concentration of spectral power around the seasonal frequency that determines the extended intervals of instability. Indeed, the maximum value of the amplitude always occurs during the period of positive $\delta$. In all these cases the interpretation qualitatively follows from simple, quasi-steady ideas about the instability. Growth occurs when the shear exceeds the classical critical value and the nonlinearity acts to yield periodic or chaotic finite-amplitude solutions, depending on the degree of supercriticality and dissipation. The principal physical point of interest is the fact that this behaviour is observed in a parameter range in which the time-averaged shear is stable. If one were to employ the time-averaged shear as the basic state the wave solutions would decay away to zero. It is the 'seasonal' excursion into temporary 

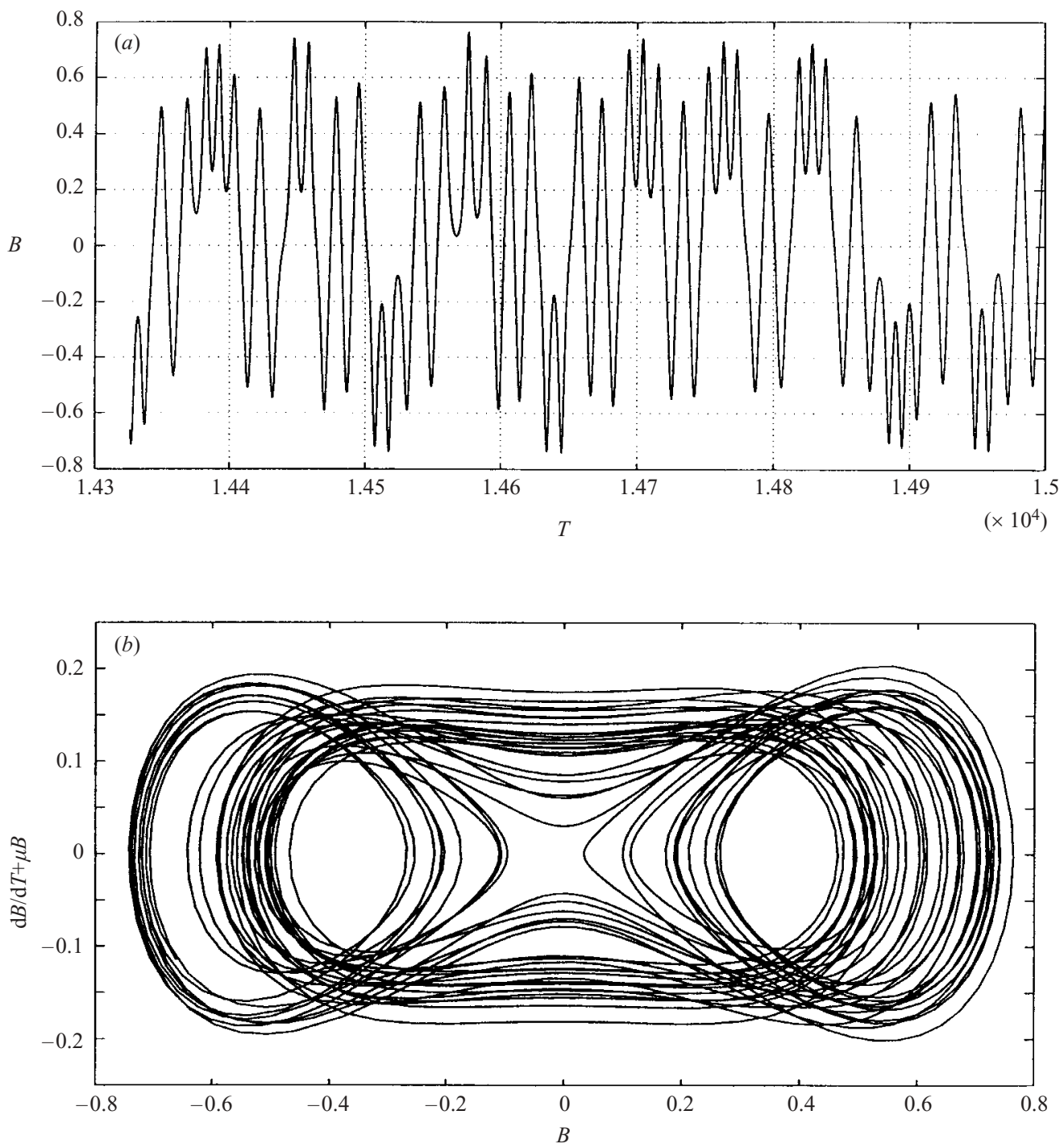

FiguRE 6. As in figure 4 except that $H=0.4$ and $\mu=0.001$. The solution is clearly aperiodic.

(a) Time history for the last $95 \%$ of the run. (b) Phase plane for the same time interval.

instability that allows the persistent presence of the unstable waves. The situation is even more interesting when both the time-averaged shear and its instantaneous value are always subcritical with respect to the classical criterion, a situation described in $\S 6$.

\section{Nonlinear dynamics and parametric instability $G<0, H<1$}

When $G=-1$ and $H<1$ the supercriticality $\delta$ is always negative and conventional application of the stability conditions would suggest that the flow remains stable to baroclinic disturbances. This turns out not to be the case for, as we have noted in $\S 3$, the possibility of parametric instability can destabilize the shear flow in this 

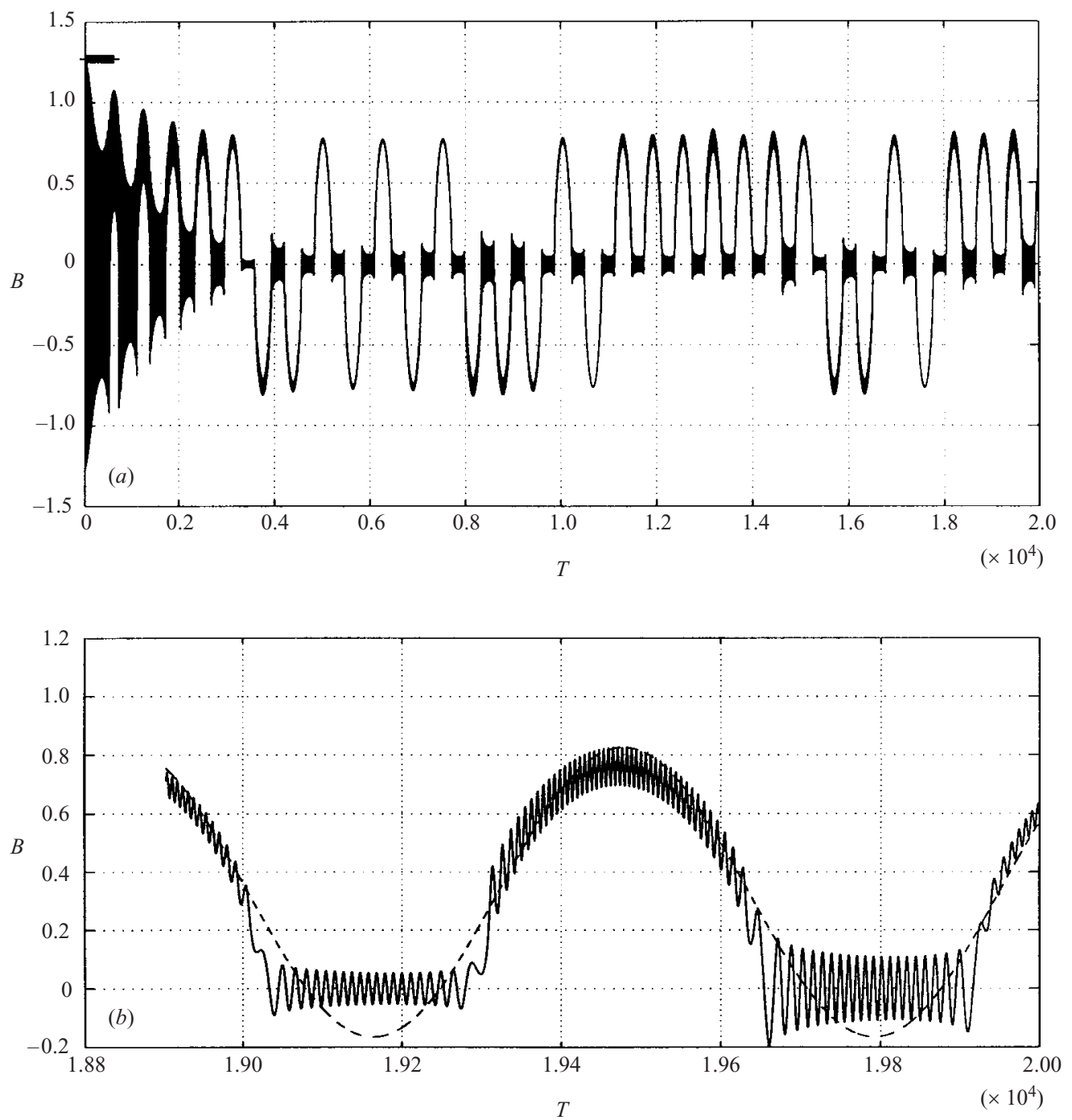

FigurE 7. The aperiodic solution for $G=1, H=1.5, \mu=0.001, \omega=0.01$. (a) The complete time history. (b) A detail of the last $5 \%$ of the calculation. The dashed curve indicates the overall value of the supercriticality. For small $\omega$ the solution grows during periods of positive $\delta$.

parameter range. Moreover, as $H$ increases from zero, the range of frequencies for which parametric instability occurs broadens rapidly. In this section we take up the question of the nonlinear dynamics of the baroclinic system in this parameter range.

Returning to the fundamental equations $(3.13 a, b)$ it is first useful to consider an analytical, asymptotic solution that turns out to be reasonably accurate rather far beyond its formal range of validity. We suppose that $H$ in $(3.13 a)$ is small and write

$$
H=\gamma h, \quad \gamma \ll 1
$$




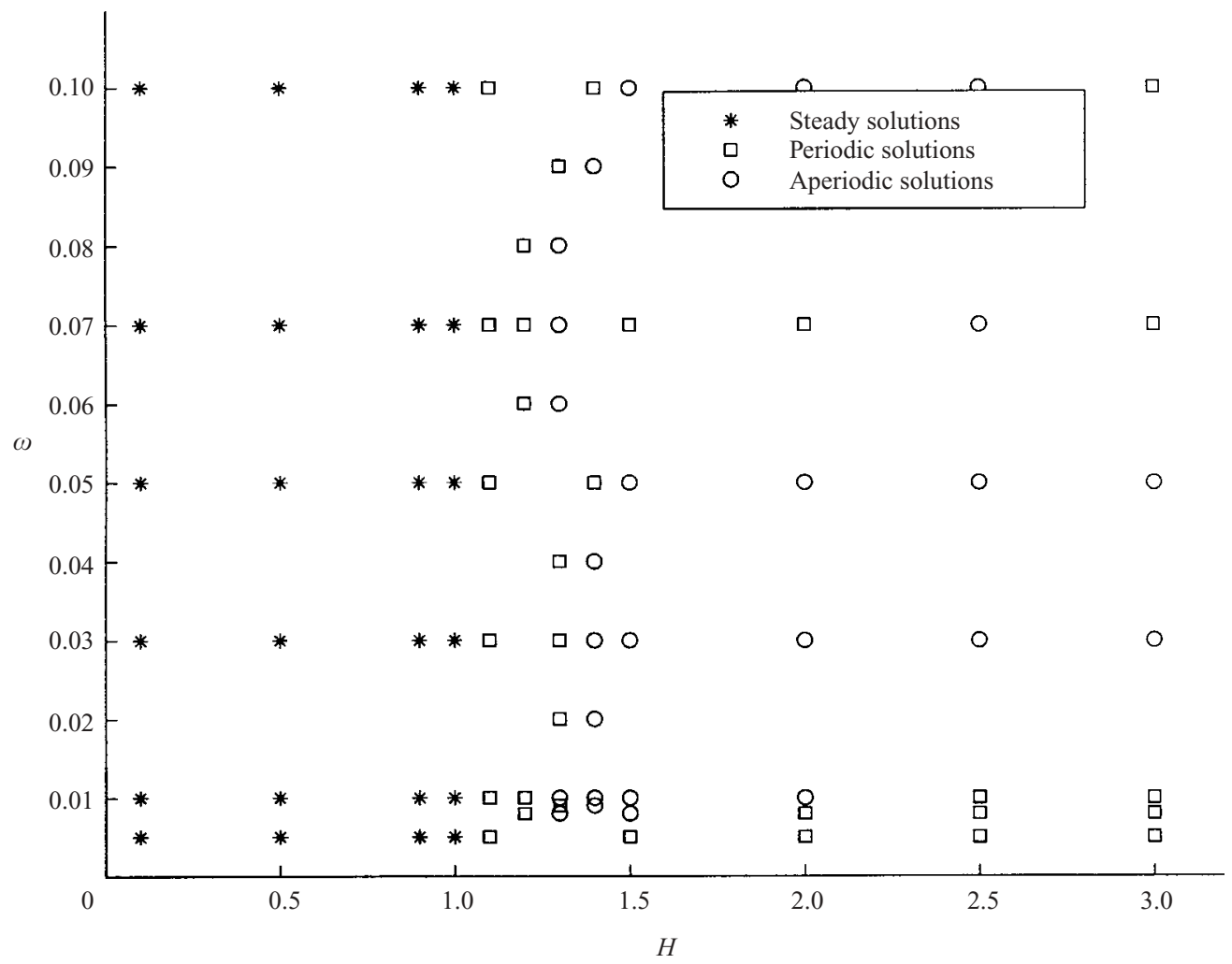

Figure 8. A regime diagram for $G=-1$ in the $(H, \omega)$-plane. Values of $\omega$ are restricted to values $<0.1$. Larger $\omega$ are discussed in $\S 5$. Squares denote periodic solutions while open circles denote aperiodic solutions. Asterisks denote solutions that tend to zero for large time.

We recall that $H$ already represents a small increment in the shear so (6.1) is a further asymptotic perturbation. We introduce the amplitude parameter $\eta=\gamma^{1 / 2}$ and write

$$
\begin{aligned}
& B=\eta\left[B_{0}+\eta B_{1}+\eta^{2} B_{2}+\cdots\right], \\
& P=\eta^{2}\left[P_{0}+\eta P_{1}+\eta^{2} P_{2}+\cdots\right] .
\end{aligned}
$$

For the purposes of this expansion we assume $\mu=O\left(\gamma^{1 / 2}\right)$. We define, as before,

$$
\sigma=\left(-2 r^{2} G\right)^{1 / 2}>0 \quad \text { (and real). }
$$

Inserting the expansions $(6.2 a, b)$ into $(3.13 a, b)$ results at lowest order in

$$
\begin{gathered}
\frac{\mathrm{d}^{2} B_{0}}{\mathrm{~d} T^{2}}+\sigma^{2} B_{0}=0, \\
\frac{\mathrm{d} P_{0}}{\mathrm{~d} T}=\frac{\mathrm{d} B_{0}^{2}}{\mathrm{~d} T},
\end{gathered}
$$

whose solutions are

$$
\begin{gathered}
B_{0}=a \cos \sigma T+b \sin \sigma T \\
P_{0}=a^{2}(1+\cos (2 \sigma T)) / 2+b^{2}(1-\cos (2 \sigma T)) / 2+a b \sin (2 \sigma T)+\text { const. }
\end{gathered}
$$



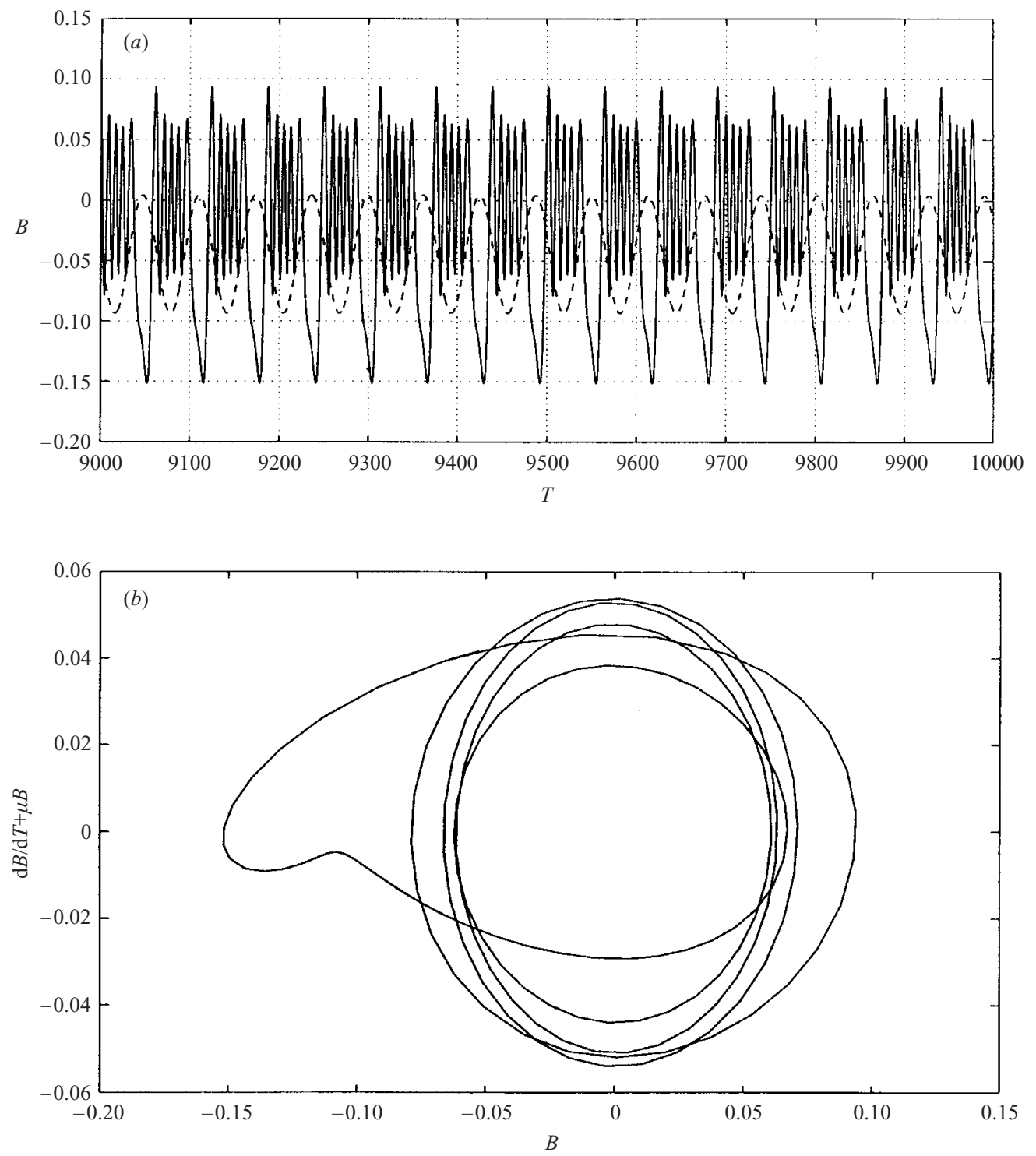

Figure 9. The solution for $G=-1, H=1.1, \mu=0.01, \omega=0.1$. The time-averaged shear is stable and the flow experiences brief periods of supercriticality. (a) Time history of the solution. Note the dashed curve, indicating that $\delta$ is greater than zero only for small intervals of time. (b) Phase plane indicating the periodicity of the solution.

The constant in $(6.5 b)$ can be determined from the integration of $(3.13 b)$ over a period of the solution, namely,

$$
\bar{P}_{0}=2 \overline{B_{0}^{2}}
$$

where an overbar represents an integral over a period of the solution $2 \pi / \sigma$. This determines the constant as

$$
\text { const. }=\frac{a^{2}+b^{2}}{2},
$$



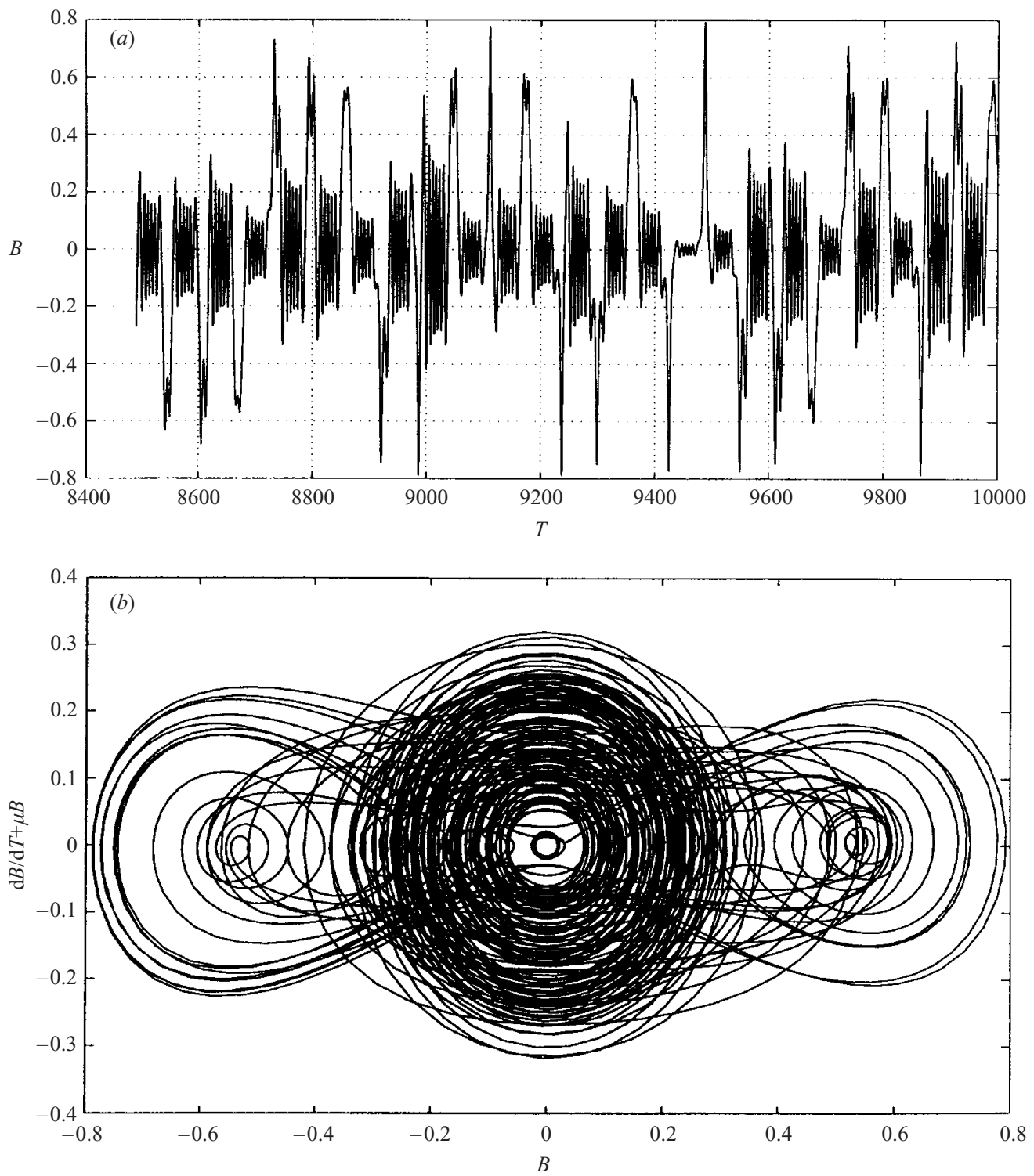

Figure 10. As in figure 9 except that $H=2.2$. The solution is now aperiodic.

so that

$$
P_{0}=\left(a^{2}+b^{2}\right)+\left(a^{2}-b^{2}\right) \cos (2 \sigma T) / 2+a b \sin (2 \sigma T) .
$$

At next order we find simply that $B_{1}=0$ while at $O(\gamma)$ we obtain the following two equations as solvability conditions:

$$
\begin{aligned}
& 2 \frac{\sigma \mu}{\eta^{2}} a-r^{2} \frac{\gamma}{\eta^{2}} h-\frac{5}{4} b\left(a^{2}+b^{2}\right)=0, \\
& 2 \frac{\sigma \mu}{\eta^{2}} b-r^{2} \frac{\gamma}{\eta^{2}} h+\frac{5}{4} b\left(a^{2}+b^{2}\right)=0,
\end{aligned}
$$



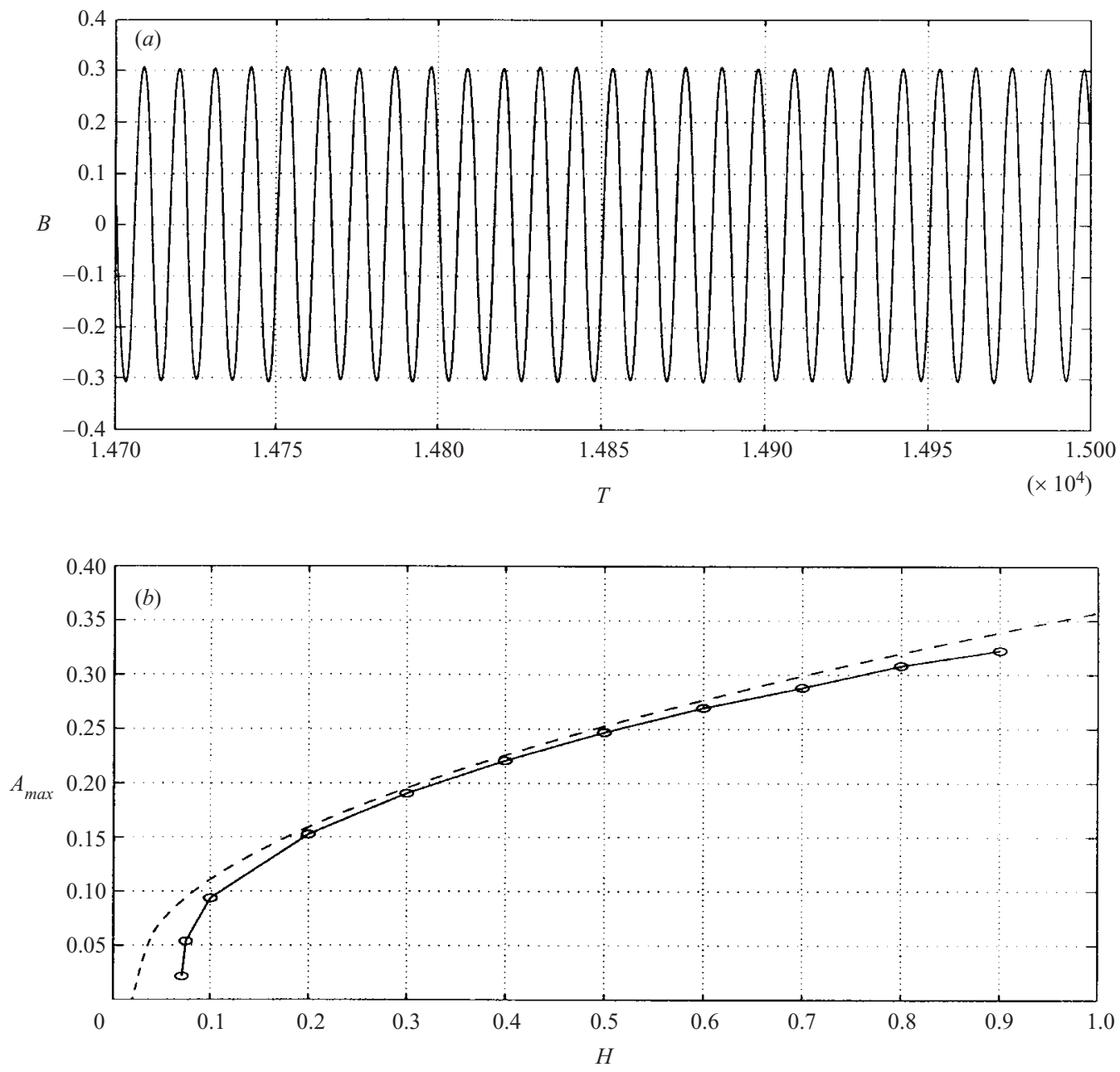

FiguRE 11. For the subcritical parametric instability at $\omega=1.1303, \mu=0.01, G=-1$. (a) The periodic solution for $H=0.8$. (b) A comparison of the analytical prediction given by $(6.11 a)$ (dashed curve) and the direct integration of $(3.13 a, b)$, indicated by the circles and joined by the solid line.

as well as the standard Mathieu condition $\omega=2 \sigma$. We shall subsequently relax this latter condition.

Writing

$$
a=R \cos \theta, \quad b=R \sin \theta,
$$

leads to the solution

$$
\begin{gathered}
\eta R=\frac{2 r}{5^{1 / 2}}\left[(\gamma h)^{2}-\frac{\mu^{2} \omega^{2}}{r^{2}}\right]^{1 / 4}, \\
\sin (2 \theta)=\frac{\omega \mu}{r^{2}(\gamma h)} .
\end{gathered}
$$

Figure 11 shows a solution for $H=0.8$, which is not small according to the scaling apparently required by (6.1). However, the solution is periodic as predicted with an 


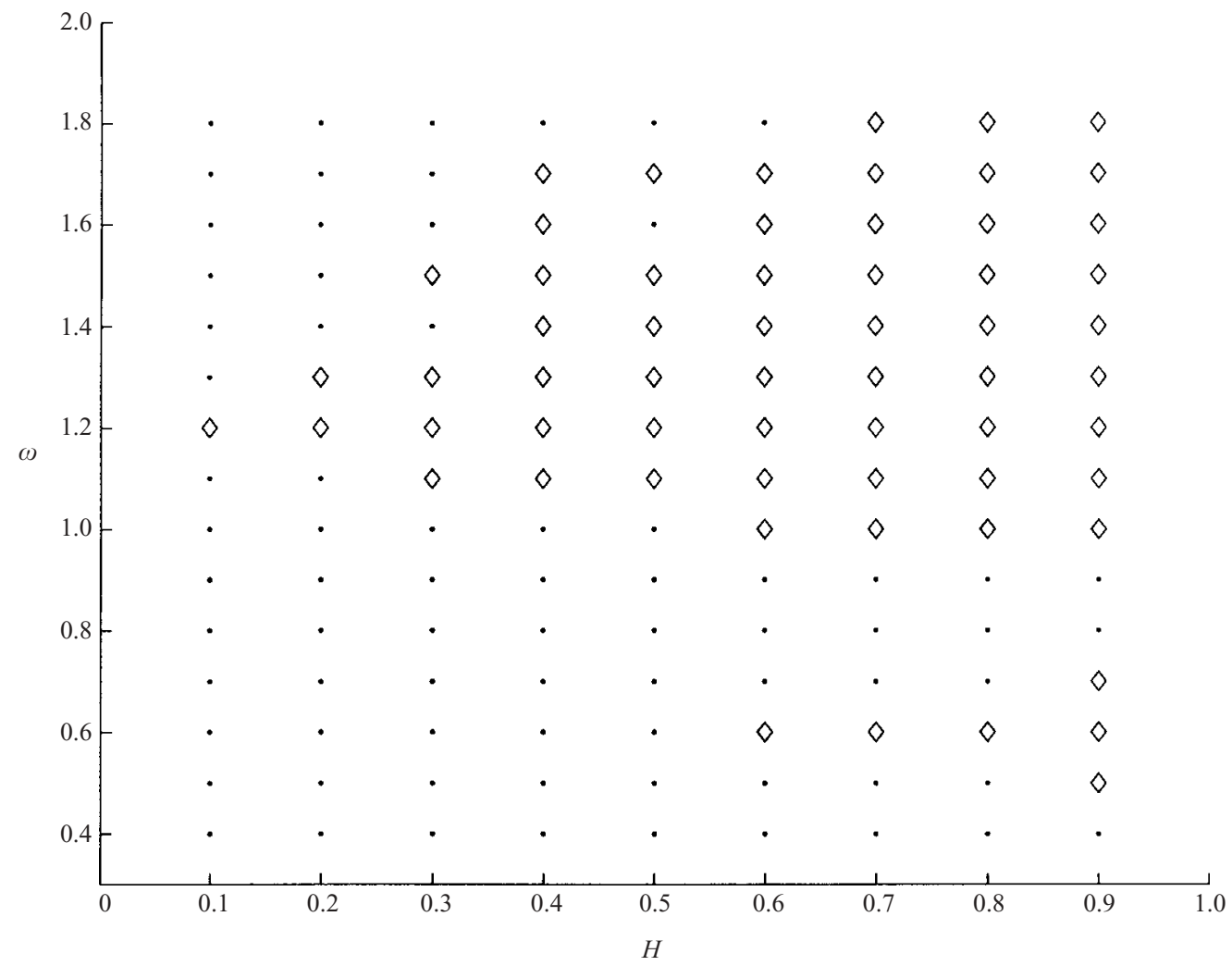

FIgURE 12. The regime diagram for $G=-1, H<1$ for $\omega$ in the range of the frequencies subject to parametric instability. The diamonds denote periodic solutions and the small dots solutions with zero amplitude.

amplitude for $B$ that is around 0.32 i.e. $O(1)$ but very close to the prediction of $(6.11 a)$. The agreement between the asymptotic result given by $(6.11 a)$ and the full numerical results is shown in figure $11(b)$. The agreement is seen to be very good even up to the limiting value $H=1$. Similar results with good agreement between analytic and numerical results have been obtained at the next lowest critical Mathieu frequency $\omega=O(0.6)$. In fact, as $H$ increases, the frequency range over which parametric instability occurs increases, with consequent finite-amplitude periodic oscillations of the limit-cycle type found over a larger and larger range of $\omega$. It is important to note that the amplitude attained by the disturbance due to parametric instability is of the same order as the equilibrated amplitude of the steady, classical problem (compare figure 11 and figure 2). Figure 12 shows the domain of periodic solutions. Positions in the plane where finite-amplitude periodic solutions are found are indicated by an open diamond. Two regions are evident; each broadens out in $\omega$ for increasing $H$. The dominant solution at $\omega=2 \sigma$ is periodic with frequency $\sigma$ and the harmonics $3 \sigma$. A somewhat weaker response occurs at $\omega=\sigma$ and is periodic with frequency $\sigma$ and the $2 \sigma$ and $3 \sigma$ harmonics. For each mode the range of $\omega$ in which the instability is found eventually widen to include a large band of frequencies of $O(\sigma)$.

We have calculated the numerical solutions of $(3.13 a, b)$ over a range of frequency for $H=0.2$ near the Mathieu frequency $\omega=1.1303$ (for $K=(2.1 F)^{1 / 4}$ ) and have found the solutions to be dependent on initial data. For all the calculations described 


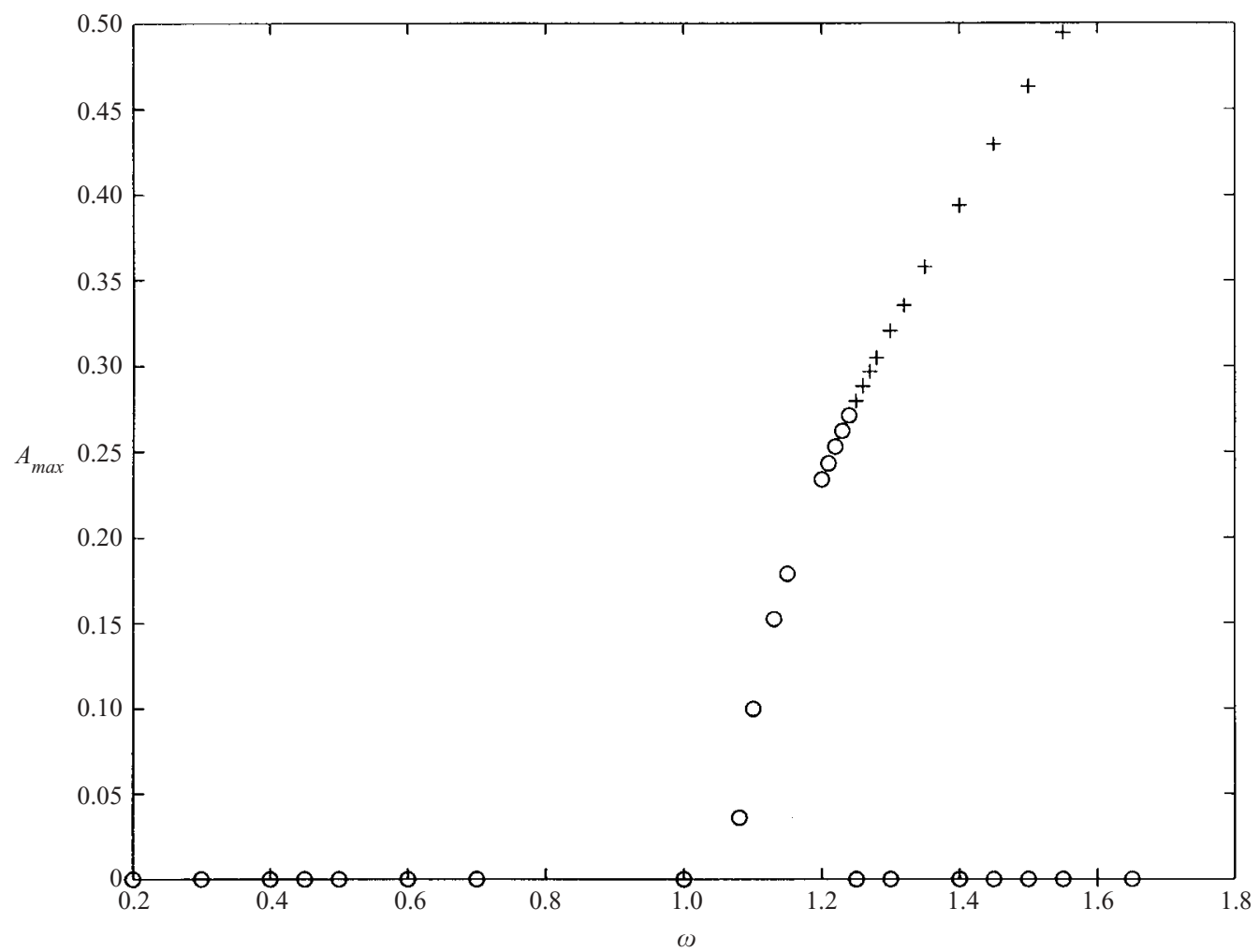

FIGURE 13. The amplitude of the periodic solution for $H=0.2$ as a function of $\omega$ for the dominant parametric instability. $G=-1, \mu=0.01$. The open circles are numerically generated with the standard initial data as described in the text. The plus signs indicate the amplitude of the periodic solution found by using as initial conditions the values of the variables from the calculation at slightly smaller $\omega$. A region in $\omega$ in which the solution is multi-valued is evident.

in this paper our initial conditions have been

$$
[B(0), \mathrm{d} B / \mathrm{d} T(0)+\mu B(0), P(0)]=[0.1,0.1,0] .
$$

In doing so, we obtained the following results for the wave amplitude of the periodic solutions as shown in figure 13. The open circles are the amplitudes of the periodic solutions obtained with the standard initial conditions. As $\omega$ increases above $\sim 1.24$ there is an abrupt crash in the amplitude of the oscillation. For these initial conditions the solution above that value of $\omega$ always tends to zero. If, however, one starts the calculation with initial conditions corresponding to the end-state values of the variables in the periodic solution at a slightly smaller $\omega$ the solution continues as a periodic solution with a larger amplitude as shown by the + signs in figure 13 . Above a certain value, which is $\omega \approx 1.5$ the periodic solution is no longer found. There appears therefore to be a range of $\omega(1.24<\omega<1.55)$ in which there are two possible end states, either a wave-free state or a finite-amplitude oscillation. In the usual fashion such multi-valued response curves indicate a hysteresis in the system's response as the frequency is altered in this range, depending on whether the frequency is increasing slowly from below this interval, in which case the upper branch of the response curve would be followed or, if the frequency is slowly decreasing from above this interval, the system moves along the lower (zero-amplitude) branch of the 
curve. This implies rapid transitions between the two branches at the limits of the multi-valued region.

We have tried to investigate the behaviour analytically by the method leading to (6.11) by allowing the frequency to vary as

$$
\omega=2 \sigma+\varpi, \quad \varpi \ll 2 \sigma,
$$

where here $\varpi$ is a small correction to the frequency of the oscillating shear.

Essentially repeating the analysis which led to (6.11) we now obtain

$$
\eta R=\frac{2}{5^{1 / 2}}\left[\left(r^{2}(\gamma h)^{2}-4 \sigma^{2} \mu^{2}\right)^{1 / 2}+\varpi / \sigma\right]^{1 / 2} .
$$

This result qualitatively matches the upper branch in figure 13 but does not yield an upper limit in frequency to the upper branch of the response curve. Of course, we do not expect (6.13) to be valid for $\varpi / \sigma=O(1)$ but we have no explanation for the discrepancy between (6.13) and the numerical result which seems to yield a definite upper limit to the frequency range for the upper branch.

The most important result in this parameter range is the existence of finite-amplitude waves in spite of the fact that the shear is, at every moment, subcritical with respect to the classical theory of baroclinic instability. It is due entirely to the parametric instability provoked by the time-dependent shear of the basic state. Since realistic atmospheric and oceanic flows are never completely steady this would seem to suggest a larger domain of instability than is usually recognized by classical normal-mode theory for steady flows.

\section{Summary and discussion}

The baroclinic instability of the two-layer model on the beta-plane in the classical model of Phillips (1954) has been extended in the present study to include the effects of time-varying baroclinic shear. The linear problem in the neighbourhood of the classical threshold of instability has the properties of the well-known Mathieu equation with the accompanying phenomena of the stabilization of supercritical shears and the destabilization of subcritical shears.

The focus of the paper, instead, is on the nature of the finite-amplitude behaviour of the baroclinic waves in the neighbourhood of the threshold when the vertical shear of the flow is a periodic function of time. By choosing a simple dissipation mechanism, the damping of perturbation potential vorticity, we can identify unsteady final states with the presence of the oscillating shear.

For slightly supercritical shears we find that the presence of the oscillating shear can lead to irregular oscillatory wave amplitudes and a broad enough spectrum (in frequency) to allow a characterization of the flow as chaotic. Since the parameter space of even this idealized problem is so large (four-dimensional) it has not been possible to survey the behaviour very finely, but roughly speaking the stronger the periodic component of the shear the more likely aperiodic behaviour is.

When the steady part of the shear is subcritical the problem neatly divides in two parts. For oscillating shears strong enough to 'lift' the shear into the unstable region, even briefly, the wave amplitude dynamics can become aperiodic. Each episode of temporary supercriticality allows finite-amplitude growth that decays sharply as the shear sinks below the critical threshold. Enough of the disturbance remains to be excited by the next 'season of instability' but the generally incommensurate periods of the seasonal cycle and the more rapid oscillations due to nonlinearity during the 
season of stability generally ensures that the start of each new epoch of instability possesses different initial conditions thus leading to aperiodic behaviour. We stress that this interesting finite-amplitude growth and oscillation occurs, in this case, when the time-averaged shear is subcritical.

Even when the oscillating part of the shear is not strong enough to lift the shear into the classical supercritical region, parametric instability can destabilize the flow. This occurs even though at each instant the flow is subcritical. The parametric instability in finite amplitude leads to a periodic solution with the period of the Mathieu-like instability and its nonlinear harmonics. Our calculations have shown a direct dependence of the amplitude of the disturbance on the size of the oscillating component of the shear. We have also found, as shown in $\S 6$, that for a range of frequencies, two solutions are possible. Either the wave eventually decays leaving a subcritical state impervious to further disturbance, or a finite-amplitude periodic oscillation can occur. The multiple states suggest the existence of hysteresis effects in the wave amplitude as a function of frequency in this subcritical region.

We emphasize that the behaviour of the system depends on the periodicity of the basic state and does not require that the periodicity be harmonic, although we have used that simplification in our calculations. Figure $14(c)$ shows the response of the system for $G=-1$ and for $H(t)$ as shown in figure 14(a). The Fourier amplitudes of the cosine series representing $H(t)$ is shown in figure $14(b)$ for the series of figure $14(a)$ :

$$
H=\sum_{n=1}^{50}\left(\frac{2}{n \pi}\right)\{\sin (n \pi / 2)-\sin (3 n \pi / 2)\} \mathrm{e}^{-0.1 n} \cos (n \omega T) .
$$

The amplitude of $H$ is then multiplied by 0.52 to keep $H<1$ for all $t$. The response as shown in figure $14(c)$ is similar to the purely harmonic case of figure 11 except that the presence of higher harmonics in $H(t)$ now yields a mildly aperiodic amplitude behaviour.

It is straightforward to show that all the instabilities discussed here satisfy the usual energy budget analysis (e.g. Pedlosky 1987) so that the perturbation energy is derived from the usual eddy fluxes of thickness (heat) as in the baroclinic problem. The question remains though, as to how this energy can be released while the flow, as in $\S 6$, is always subcritical to baroclinic instability.

It is illuminating in this regard to restate one of the conditions for instability (Pedlosky 1987). For the two-layer model in the absence of horizontal shear,

$$
\frac{\partial}{\partial t}\langle E\rangle-\sum_{i=1}^{2} \frac{\partial \eta_{i}^{2}}{2 \partial t} U_{s} \frac{\partial Q_{i}}{\partial y}=0
$$

In (7.1) $\langle E\rangle$ is the total integrated perturbation energy, $U_{s}$ is the shear, $\partial Q_{i} / \partial y$ is the potential vorticity gradient in the $i$ th layer and $\eta_{i}$ is the meridional displacement in layer $i$. Since both the shear and the potential vorticity gradients are functions of time, (7.1) cannot be written as a conservation statement, rather

$$
\frac{\partial}{\partial t}\left\lfloor\langle E\rangle-\sum_{i=1}^{2} \frac{\eta_{i}^{2}}{2} U_{s} \frac{\partial Q_{i}}{\partial y}\right\rfloor=-\sum_{i=1}^{2} \frac{\eta_{i}^{2}}{2} \frac{\partial}{\partial t}\left(U_{s} \frac{\partial Q_{i}}{\partial y}\right) .
$$

When the shear is independent of time, the right-hand side of (7.2) vanishes and the term in the square bracket on the left-hand side can be rendered positive definite when the flow is subcritical by the addition of a steady barotropic zonal flow to which the problem is (Galilean) invariant. The added term on the right-hand side of 

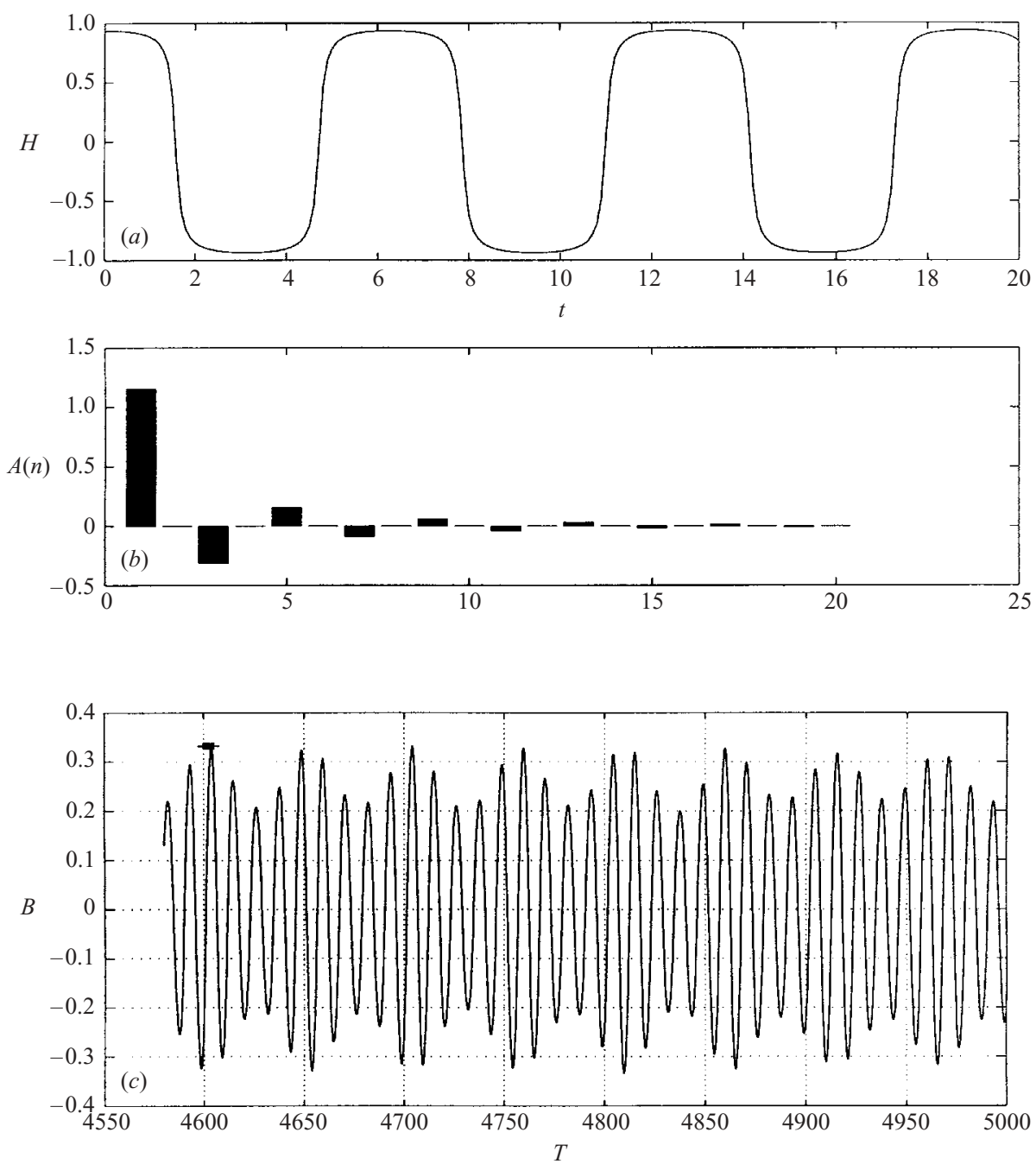

FIgURE 14. An example of the response for $G<0, H<1$ of parametric instability for a periodic $H(t)$ which is not harmonic. (a) The function $H(t)$ is periodic and nearly a series of step functions. ( $b)$ The amplitude $A(n)$ of the cosine series representing $H(t)$. (c) The response of the perturbation amplitude as a function of time. The response is qualitatively similar to the response of figure 11 but the added harmonic content of $H(t)$ has rendered the solution aperiodic.

(7.2) when the flow is time dependent is the 'pumping' term, familiar from simple dynamical problems leading to Mathieu-like instability, that is responsible for the growth of the pseudo-energy on the left-hand side of (7.2) even in cases when the flow is subcritical. Since the time-dependent shears considered here are weak variations around a steady flow the terms $U_{s} \partial Q_{i} / \partial y$ will be dominated by a term oscillating with $\omega$. The disturbance field $\eta_{i}$ oscillating with frequency $\sigma$ will produce a steady forcing of the energy if $\omega=2 \sigma$, the principal Mathieu resonance condition. This is the basic mechanism for the instability. Note, however, that such an exact 2:1 tuning is only necessary when the oscillating part of the current is relatively small. 
Thus, the presence of time-varying shear, which we think of as a highly idealized model for seasonal variations in the background state of atmospheric and oceanic currents can itself induce instabilities for levels of shear hitherto considered stable.

The authors would like to express their gratitude to Xavier Carton for helpful discussions on the linear aspects of this problem. We are also grateful for useful discussions with Glenn Flierl, Francis Poulin and Carl Wunsch and the comments of several anonymous reveiwers. J.P.'s research is supported in part by a grant from NSF, OCE 9901654. J. T. grateful acknowledges support from both the same NSF grant and the MIT/WHOI Joint Program.

\section{Appendix. The coefficient $N$ in (3.11)}

After solving (3.9) subject to the condition that $\partial \Phi_{T} / \partial y$ vanish on $y=0$ and $y=1$, insertion in (3.8b) yields the ordinary differential equation (3.11). After considerable algebra it is straightforward to show that

$$
N=\frac{2 m_{p}^{2} F k^{2}\left(K^{2}-F\right)}{K^{2}\left(2 F+K^{2}\right)}\left[\frac{1}{2}-\frac{\left(K^{4}-2 F^{2}\right)}{\left(4 m_{p}^{2}+2 F\right)\left(K^{2}-F\right)}\left\{\frac{1}{2}+\frac{4 m_{p}^{2} \tanh (F / 2)^{1 / 2}}{\left(4 m_{p}^{2}+2 F\right)(F / 2)^{1 / 2}}\right\}\right]
$$

\section{REFERENCES}

Cole, J. 1968 Perturbation Methods in Applied Mathematics. Blaisdell, Waltham, MA.

Davis, S. H. \& Rosenblat, S. 1980 A quasiperiodic Mathieu-Hill equation. SIAM J. Appl. Maths 38, $139-155$.

von Kerczek, C. 1982 The instability of oscillatory plane Poiseuille flow. J. Fluid Mech. 116, 91-114.

Klein, P. \& Pedlosky, J. 1992 The role of dissipation mechanisms in the nonlinear dynamics of unstable baroclinic waves. J. Atmos. Sci. 49, 29-48.

Majda, A. J. \& Shefter, M. G. 1998 Elementary stratified flows with instability at large Richardson number. J. Fluid Mech. 376, 319-350.

Morse, P. M. \& Feshbach, H. 1953 Methods of Theoretical Physics, Part 1. McGraw-Hill.

Pedlosky, J. 1982 A simple model for nonlinear critical layers in a baroclinic wave. J. Atmos. Sci. 39, 2119-2127.

Pedlosky, J. 1987 Geophysical Fluid Dynamics. Springer.

Phillips, N. A. 1954 Energy transformations and meridional circulations associated with simple baroclinic waves in a two-level, quasi-geostrophic model. Tellus 6, 273-286.

Poulin, F. J., Flierl, G. R. \& Pedlosky, J. 2003 Parametric instability in oscillatory shear flow. J. Fluid Mech. 481, 329-353.

QIU, B. 1999 Seasonal eddy field modulation of the North Pacific Subtropical Countercurrent: TOPEX/ Poseidon observations and theory. J. Phys. Oceanogr. 29, 2471-2486.

Rosenblat, S. 1968 Centrifugal instability of time dependent flows. Part 1. Inviscid periodic flows. J. Fluid Mech. 33, 321-336.

Zounes, R. S. \& RAND, R. H. 1998 Transition curves for the quasi-periodic Mathieu equation. SIAM J. Appl. Maths 58, 1094-1115. 